\title{
THE EFFECT OF LEARNING CONDITION ON MEMORY FOR AND INTEGRATION OF RELATED INFORMATION
}

\author{
by \\ RENNIE KENDRICK
}

\begin{abstract}
A THESIS
Presented to the Department of Biology and the Robert D. Clark Honors College

in partial fulfillment of the requirements for the degree of Bachelor of Science
\end{abstract}

June 2020 


\section{An Abstract of the Thesis of}

Rennie Kendrick for the degree of Bachelor of Science

in the Department of Biology to be taken June 2020

Title: The effect of learning condition on memory for and integration of related information

\section{Approved: _ Dr. Dagmar Zeithamova-Demircan Primary Thesis Advisor}

Approved: Dr. Peter Wetherwax

\section{Biology Honors Faculty Representative}

Although we often are tasked with learning pieces of information that are related in some way in educational settings, the learning conditions that promote learning of and connections across related information are still poorly understood. In this thesis, I asked: Which instruction methods promote learning of and connections across (integration of) related information? In the two experiments in this thesis, participants were presented related information in two different formats, and we assessed their memory for related information, in addition to how well they could integrate related information to derive new knowledge. The two presentation formats incorporated the same number of exposures of related information, but the sequence with which information was presented was manipulated: In one presentation format (blocked) participants strongly learn one set of information, before being confronted with a related set of information, whereas participants learn related information in parallel in the other (interleaved). In Experiment 1, we found that blocked presentation enhanced both memory for and integration of related information. However, given potential effects of 
testing order on our results, we more closely examined memory for related information in Experiment 2. In Experiment 2, we no longer found a difference in overall memory for related information between the two presentation formats. In fact, we found evidence that presence of shared information enhanced learning of related information after interleaved presentation only. Regardless, the results of both experiments demonstrated that manipulating just the sequence with which information is presented, without increasing the number of exposures, can enhance learning, an impactful finding in an era of increased pressures to maximize instructional time. 


\section{Acknowledgements}

I would like to thank Professor Dasa Zeithamova for helping me solidify my

ideas into two concrete, interesting, and fulfilling experiments. Dr. Zeithamova allowed me to see an independent project from start to finish, an extremely rare and fulfilling opportunity to have as an undergraduate. I would also like to thank PhD student Ms. Lea Frank for her extremely thorough edits on the thesis draft, and for her continual support on this project. Both Dr. Zeithamova and Ms. Frank were fantastic mentors who taught me so much about experimental design, statistical analysis, and writing. I also would like to thank my Clark Honors College Representative, Dr. Mark Carey for his thorough comments on a draft and Dr. Peter Wetherwax from the Biology Deparmtent for serving on my Honors Thesis Committee. I also would like to thank my parents, Eliza Coblentz and Mike Kendrick for their constant and unwavering support of me in my educational endeavors—-they have helped me through so many academic and nonacademic challenges. I would also like to thank my sister, Parkes Kendrick, who always can cheer me up over the phone or talk me through any problem. 


\section{Table of Contents}

Introduction 1

Hypotheses $\quad 9$

Implications 11

Methods: Experiment $1 \quad 13$

Results: Experiment 1

Discussion: Experiment $1 \quad 25$

Methods: Experiment 2

Results: Experiment 2

Discussion: Experiment 2

$\begin{array}{ll}\text { Discussion } & 50\end{array}$

Conclusions $\quad 59$

Supplementary Figures $\quad 61$

$\begin{array}{ll}\text { Experiment } 1 & 61\end{array}$

Experiment $2 \quad 63$

Supplementary Tables $\quad 66$

$\begin{array}{ll}\text { Bibliography } & 67\end{array}$ 


\section{List of Figures}

Figure 1. Overview of Experiment 1 design 18

Figure 2. Accuracy on associative inference test 19

Figure 3. Accuracy on associative inference test trials for which participants correctly $\begin{array}{ll}\text { answered corresponding direct test trials } & 20\end{array}$

Figure 4. Mean accuracy and RT on direct tests, grouped by presentation group (outliers included) $\quad 24$

Figure 5. Overview of Experiment 2 design $\quad 37$

Figure 6. Mean accuracy on direct tests 40

Figure 7. Mean RT on direct tests $\quad 42$

Figure 8. Effect of association class and presentation order on test RT 43

Figure S1. RT on associative inference tests 61

Figure S2. Mean accuracy and RT on direct tests, grouped by presentation group (outliers excluded)

Figure S3. Mean accuracy on direct tests (outliers excluded) 63

Figure S4. Mean RT on direct tests (outliers excluded) 64

Figure S5. Associative inference test accuracy 65

Figure S6. Associative inference test RT 65 


\section{List of Tables}

Table 1. Mean accuracy (and SD) on direct tests 21

Table 3. Mean accuracy (and SD) on direct tests 40

Table 4. Mean RT (and SD) on direct tests 41

Table S1. Mean accuracy (and SD) on direct tests (outliers excluded) 66

Table S2. Mean RT (and SD) on direct tests (outliers excluded) 66 


\section{Introduction}

Memory extends beyond the simple recollection of past events by allowing us to connect related pieces of information learned at different times to infer new knowledge. Such a memory process integrates (i.e., combines) related memories, allowing us to infer new information beyond what is contained in each memory trace alone. For example, suppose you saw Person A walking a Dalmatian on Tuesday and then saw Person B walking that same Dalmatian on Wednesday. Integration of the two memories, based on their shared feature of the Dalmatian, might lead you to conclude that Person A and Person B are related in some way. In this case, integration of related memories allows you to infer a relationship between Person A and B, despite never seeing Person A and B together. Thus, integration can generate new information that extends beyond what is directly experienced and stored in memory.

Memory integration can also serve knowledge generation in educational settings. For example, an individual may learn a mathematical function for sine functions in calculus, and then a week later, learn in physics that light waves are sine waves. When learning about light waves in physics, the individual may recall certain characteristics about sine waves that they learned about in calculus and apply this to understand a new characteristic about light waves (e.g., that the formula that describes the shape of light waves must follow a certain form). Again, integration of memories containing related information can lead to new information beyond what was contained in each memory alone.

In fact, educators have always strived not just to promote student's retention of taught information, but to encourage students to synthesize learned material (via 
memory integration). Synthesizing learned material is necessary to answer difficult or novel questions and can increase retention of the learned material itself (Anderson and McCulloch, 1999; Kuhl et al., 2010). However, while extensive research has identified learning conditions that promote memory for isolated pieces of information (Cepeda et al., 2006; Janiszewski et al., 2003; McCallister and Ley, 1972), it is still unknown which learning conditions promote memory for and integration of related information.

To address this gap in the literature, this thesis asks: Which learning conditions promote integration of and memory for related information?

The two learning conditions compared in this thesis vary based on the level of exposure participants have to one set of information before learning a second set of related information. Because these two learning conditions differ based on how information is presented to participants during learning, I will refer to them as presentation formats. During blocked presentation, participants see repetitions of one set of information before they see the second, related set of information. During interleaved presentation, exposures of the two sets of related information are alternated.

In terms of the real-world example of a student taking physics and calculus, the study asks: Would students be more likely to integrate their calculus and physics understanding of sine waves if they strongly learned the calculus concept before the physics concept? Or, would learning the two concepts in parallel promote stronger connections across learned material?

Blocked and interleaved presentation of information are compared for three main reasons. First, blocked and interleaved presentation can both be implemented in the classroom (Rohrer, 2012) and students may commonly be presented information in 
both formats. Second, the drawbacks and benefits of these two formats of learning have been extensively studied in many domains of learning (Carvalho and Goldstone, 2014; Rau, et al., 2013; Shea and Morgan, 1979), such as category learning, but is not known how they impact memory for (Kuhl et al., 2010; Schlichting and Preston, 2014; van Kestern et al., 2018; Underwood, 1949; Whitely, 1927; Postman, 1962) or integration (Schlichting, Jeanette, and Preston 2015) of related information. And third, the literature has identified factors that enhance both memory for and integration of related information, but some factors are enhanced by blocked presentation (Schlichting and

Preston, 2014; Kuhl et al., 2010) and others by interleaved presentation (Zeithamova and Preston, 2017; Cepeda et al., 2006), necessitating research that directly compares the two presentation formats.

\section{Integration of related information}

For example, research has found that reactivation of one set of information that shares features with another set of information promotes memory integration (Zeithamova et al., 2012; Kuhl, et al., 2010; Schlichting and Preston, 2014; van Kesteren et al., 2018). Because more strongly-established information is more easily reactivated (Schlichting and Preston, 2014), this would suggest that individuals should robustly learn one set of information before being confronted with new, related information. Thus, blocked presentation may enhance memory integration more than interleaved presentation, as individuals more strongly establish one set of information before being confronted with a new, related set of information. Additionally, one study found that neural signatures of memory integration (Shohamy and Wagner, 2008; Schlichting and Preston, 2015) were present to a greater degree during blocked 
presentation than during interleaved presentation (Schlichting, Jeanette, and Preston, 2015).

In contrast, research has also shown that strongly established information can interfere with an individual's ability to integrate new and related information, suggesting that interleaved exposures may enhance memory integration (van Kesteren, et al., 2013; Shing and Brod, 2016). Another feature of interleaved presentation that could promote better memory integration is the close presentation of related information in time, due to alternation of sets of related information: Close presentation in time of related, but distinct, sets of information, has been shown to lead to stronger neural signatures of memory integration, and in turn, better performance on tests requiring memory integration (Zeithamova and Preston, 2017).

These contradictions highlight the need to directly compare the impact blocked versus interleaved presentation has on integration of related episodes contained in memory. Only one study to date has explicitly compared the effect the two presentation formats have on integration of related information, but in this study participants were performing with near-perfect accuracy so that neural signatures, and not behavioral outcomes (e.g., success on tests that require integration of related episodes), could be compared (Schlichting, Jeanette, and Preston, 2015). Because we are interested in identifying which learning conditions improve learning that requires memory integration, the current study importantly tests if reported increases in neural signatures of memory integration translate to better performance on tests that require integration of related information. 
Furthermore, because the various factors that affect integration ability (e.g., proximity in time or strength of initial memory) are differentially present in each learning condition, identification of which presentation format better promotes memory integration will resolve the question of which factors are most important to integration ability.

\section{Memory for related information}

Prior research into "spacing” effects suggests that memory for related information will be greater after interleaved presentation than after blocked presentation. Repetitions of presented information are presented farther apart in time during interleaved presentation than during blocked presentation. (Note: Not to be confused with related information, which is presented closer together in time during interleaved presentation.) As such, interleaved presentation has more temporal “spacing” between repetitions. Spacing repetitions of information has been shown to improve memory performance, suggesting that interleaved presentation would promote better learning in our study than blocked presentation (Cepeda et al., 2006; Janiszewski et al., 2003). Furthermore, one study specifically found that the amount of different information presented between repetitions of information, rather than time elapsed between repetitions, drove this learning enhancement. That is, time elapsed between repetitions was not sufficient to induce this effect, and intervening information was required for enhanced memory for presented information (McAllister and Ley, 1972). Because interleaved presentation has more intervening information between repetitions of information than blocked presentation, these findings further suggest that interleaved presentation will optimize learning. 
However, the impact blocked and interleaved presentation would have on learning of related information, as in this study, is still unknown. The presence of shared elements between learned information has unique implications for memory for that information. For example, the greater the overlap between two sets of information, the more interference, or competition, between learning of the related information (Verde, 2004; Hupbach, 2011). In this case, interference between related information could impair memory for both sets of information. That is, memory of the firstpresented set of information can interfere with learning of second-presented set of information (proactive interference). Alternatively, memory for the second-presented information set can interfere with the memory of the first-presented information set (retroactive interference).

It is unclear whether interference will be exacerbated more by blocked or interleaved presentation. Reactivating prior knowledge protects this information from interference from new, related information (retroactive interference; Hulbert and Anderson, 2020), and even can promote acquisition of related information (facilitation; Kuhl et al., 2010; Schlichting and Preston, 2014; van Kesteren et al., 2018). Furthermore, reactivation ease has been linked to strength of the memory (Schlichting and Preston, 2014), suggesting that participants may more easily reactivate information during blocked presentation and, as a result, have reduced interference or enhanced facilitation, both of which would enhance learning. However, other studies have found that strongly established knowledge can promote proactive interference by interfering with learning of related information (Underwood, 1949; Whitely, 1927; Postman, 
1962). In fact, one study that found presentation of information in a format that

emulated blocked presentation produced proactive interference effects (Postman, 1962).

\section{Memory for and integration of related information: competing or complementary abilities?}

Furthermore, by assessing the extent to which both memory for and integration of related episodes are enhanced by one presentation format over another, this study clarifies another unresolved question: Can memory for related information and integration of related information be promoted by the same learning conditions? Or, in other words, does promoting integration across related information (via a certain presentation format), come at a cost or benefit to memory for the related information itself?

A presentation format that enhances integration across related information could also enhance memory for the information itself, or, alternatively, impair memory for the information. To illustrate how these two processes might be opposed, a presentation format that promotes integration of information learned in physics and calculus class might come at a cost of forgetting information that an individual specifically learned in each class; because not all information students learned in each class will be integrated (i.e., information that was reviewed in one, but not the other, class would not necessarily be integrated), certain details might be lost. Alternatively, integration might solidify an individual's understanding of what they directly learned in physics and calculus, by generating helpful or clarifying insights, enhancing their memory of concepts reviewed in each class individually. 
In fact, previous research suggests that cognitive processes associated with memory integration can positively or negatively impact retention of learned material. One cognitive process associated with memory integration (Shohamy and Wagner, 2008; Schlichting and Preston, 2015) has been shown to enhance memory for related information (Anderson and McCulloch, 1999; Kuhl et al., 2010). In contrast, a different cognitive process associated with memory integration led to distortion of the original information contained in memory (Carpenter and Schacter, 2017; Tompary and Davachi, 2017). These two cognitive processes both integrate related information stored in memory, but differ based on if information is integrated during learning or once a test requires memory integration. Thus, depending on which cognitive process is biased within a presentation format that enhances memory integration, the presentation format that enhances integration may enhance or detriment memory for related information.

The present thesis consists of two experiments that together determined whether blocked or interleaved presentation enhanced integration of related information and if the same presentation format also enhanced memory for the related information itself. In Experiment 1, participants learned sets of information that shared elements, and their ability to integrate and remember related information was assessed. In Experiment 2, the impact of presentation format on memory for related information was more thoroughly characterized. In addition to assessing memory for related information, memory for isolated pieces of information was also assessed, to determine the extent to which the way in which information is presented modulates the effect shared information has on memory (i.e., whether shared information produces facilitation or interference in a given presentation format). 


\section{Hypotheses}

\section{Integration of related information}

I expect participants to perform better on tests requiring memory integration when related information sets are presented in blocked format: I predict that how wellestablished the initial information set is will be more important than its proximity in time to the second set of related information, especially given findings that neural signatures of integration are stronger during blocked rather than interleaved presentation (Schlichting, Jeanette, and Preston, 2015).

\section{Memory for related information}

I predict that the same presentation format that promotes memory integration will also lead to better memory for the related information; thus, I predict that blocked presentation will also promote memory for related information. I predict that memory integration and retention are not opposed, but rather mutually supportive, memory processes: Studies supporting a complementary relationship between neural signatures of memory integration and retention are more robust (Anderson and McCulloch, 1999; Kuhl, et al., 2010). Furthermore, specific features of blocked presentation also support my prediction that memory retention will be enhanced by this presentation format. I predict that the relationship between stronger prior knowledge and better acquisition of related information (Kuhl et al., 2010; Schlichting and Preston, 2014; van Kesteren et al., 2018), as well as greater protection from distortion of prior knowledge when related information is encountered (Hulbert and Anderson, 2020), will make strength of prior knowledge the most important factor that affects memory for related information. 
Because prior knowledge is more strongly established during blocked presentation, memory for related information will be enhanced by this presentation format. Finally, I predict that this enhancement in learning of related information will arise via facilitation effects, which have been linked to the strength with which information is initially established (Kuhl et al., 2010; Schlichting and Preston, 2014; van Kesteren et al., 2018). 


\section{Implications}

If participants were to have better memory for and integration of related information after blocked presentation, this would complicate the narrative that interleaved presentation is a superior presentation format in the classroom setting (Carvalho \& Goldstone, 2014; Rau, et al., 2013; Shea \& Morgan, 1979). In contrast, if participants were to have better memory for and integration of related information after interleaved presentation, this study would add to the literature by identifying another form of learning that is enhanced by interleaved presentation. Such a finding would indicate that interleaved presentation might provide some generalized benefit that extends across different forms of learning.

Furthermore, if memory for and integration of related information are enhanced by the same presentation format, this would mean that crucial factors that support integration across, as well as retention of, related memories are present in the same presentation format, while building on theories that cognitive processes that promote integration of related information also enhances memory for that related information (Anderson and McCulloch, 1999; Kuhl et al., 2010). If memory retention and integration are not enhanced by the same presentation format, this would suggest that crucial factors that support memory for related information are represented in one presentation format, and crucial factors that support integration are represented in the other; such a finding would provide preliminary evidence that, at least during blocked and interleaved presentation, cognitive processes associated with memory integration do not enhance memory for the related information itself (Carpenter and Schacter, 2017). However, such a finding would not necessarily mean that memory for and integration of 
related information cannot be optimized by the same presentation format per se, but that further investigation would be needed to tailor presentation formats that include crucial factors necessary for optimization of both types of learning.

Thus, either finding would contribute to our understanding of the factors that impact both memory for and integration of related information. Careful examination of which presentation formats enhance each memory process will contribute to our understanding of these cognitive processes, in addition to building on literature examining the benefits and drawbacks of blocked and interleaved presentation.

Regardless of which presentation format enhances memory integration and if the same or a different presentation format enhances memory for related information, any performance difference on tests of memory for or integration of related information between presentation formats would be significant. Such a result would indicate that memory for and integration of learned material can be enhanced simply by changing the sequence in which information is presented to individuals, without a concordant increase in the number or duration of exposures to information. This would have particularly important implications for educational settings, as maximizing instructional time has been a longstanding goal of educational research (Wang, 1979; Dolton, et al., 2003; Johnes, et al., 2017). Furthermore, instructional methods that enhance learning without increases in instructional time are especially important in our current era of increased budget cuts and reduced instructional time (Leachman, et al., 2017; Edenfield, 2013). 


\section{Methods: Experiment 1}

\section{Participants}

100 volunteers participated in this experiment. Participants received research credit in their courses for participation in the study. Four participants did not finish the study, one participant took the study with a program error (fixed for all subsequent participants), and two participants misunderstood study premises, and thus were excluded ( $n=7$ total). Participants were determined to be outliers if their direct test accuracy was greater than 2.5 standard deviations from the mean and lower than chance (0.33); $\mathrm{n}=4$ participants met this criterion. Because inclusion of these outliers $(n=4)$ significantly changed conclusions, subsequent analyses reported results with outliers included $(n=94)$ and with outliers excluded $(n=89)$.

\section{Materials}

Stimuli consisted of 234 color photographs of everyday objects.

\section{Memory task}

Stimuli were randomly organized into 156 object-object pairs (associations) such that every object-object association shared an object in common with one other association; two representative associations are shown in Figure 1A. Object associations were divided evenly into two groups (78 associations per group). Within a group, object associations did not share objects in common with any other associations. However, each object association in one group shared an object in common with a corresponding object association in the other group. 
Participants viewed six sets of object-object associations. Each set was comprised entirely of associations from one of the two groups (i.e., contained 78 associations) and sets were separated by an opportunity for participants to take a break. The group of associations presented in the first set were called AB (first-presented) associations. The group of associations presented second to participants were called BC (second-presented) associations. All participants viewed a total of three sets comprised of $\mathrm{AB}$ associations and a total of three sets comprised of $\mathrm{BC}$ associations.

The two presentation groups differed based on the sequence with which sets of $\mathrm{AB}$ and $\mathrm{BC}$ associations were presented, and are summarized schematically in Figure 1B. In the blocked presentation group, participants saw three sets comprised of the firstpresented associations ( $\mathrm{AB}$ associations) before viewing the three sets comprised of the second-presented group of associations (BC associations). In the interleaved presentation group, participants saw alternating sets of $\mathrm{AB}$ and $\mathrm{BC}$ associations. That is, the first set comprised of BC associations was presented immediately after participants viewed the first $\mathrm{AB}$ association-comprised set.

Importantly, participants in both learning conditions saw the same number of repetitions of object associations: participants saw each of the 156 associations (AB and BC associations) three times over the course of the study, and the left/right position of the $\mathrm{A}, \mathrm{B}$, and $\mathrm{C}$ stimuli were randomized across trials. $\mathrm{AB}$ and $\mathrm{BC}$ associations were randomly intermixed within a set with the constraint that the same $\mathrm{AB}$ association or corresponding $\mathrm{AB}$ and $\mathrm{BC}$ associations, which shared an object in common, were never presented back-to-back. The only potential place where these repetitions can occur is at the end of a presentation set, as the first association of the next set could be the same 
$\mathrm{AB}$ association (if the next set is also comprised of $\mathrm{AB}$ associations) or a $\mathrm{BC}$ association with the same B face (if the block is a BC block). Still, we controlled for this possibility. BC associations were also intermixed with the constraint that identical BC associations were never presented back-to-back.

Associations were presented on the screen for $3 \mathrm{~s}$ with an interstimulus interval (ISI) of $1 \mathrm{~s}$. Participants were instructed to generate a unique story or mental image linking the two objects in each association and to rate the quality of that story they generated within the $3 \mathrm{~s}$ that the association is shown on the screen by pressing a keyboard key (1 = Poor, 2 = Okay, 3 = Good, 4 = Excellent; Figure 1A). This task was designed to keep participants engaged in studying objects, and was not analyzed for the purpose of the study. Before the actual study, participants ran through a practice version of this learning and rating task in which they viewed five $\mathrm{AB}$ associations and five $\mathrm{BC}$ associations. As such, participants were aware that the associations in the study would contain overlapping information. However, participants were tested on their memory for the individual associations as a practice test, and did

In the actual study, an associative inference test was used to quantify the degree to which participants successfully could integrate related associations to derive new relationships between objects. Participants were not explicitly informed of the overlap between the $\mathrm{AB}$ and $\mathrm{BC}$ associations at the start of the experiment. Following the study portion, participants were surprised with an AC associative inference test. Directly before the associative inference test, participants were informed that $\mathrm{A}$ and $\mathrm{C}$ objects that were both associated with the same B object are indirectly related through their shared association with the B object. In the associative inference AC three alternative 
forced-choice test, participants were instructed to match an object at the top of the screen with one of three objects below that was indirectly associated with that top object, based on what they had viewed during the study portion (Figure 1C). Because participants never directly viewed A and C objects together, this test required integration of their memories for the $\mathrm{AB}$ and $\mathrm{BC}$ associations. The foil object choices were objects that were of the same object class (i.e., a C object that shared a B object in common with a different A object than the one being tested). The associative inference test was self-paced, meaning that once participants gave their response, they were advanced to the next test trial.

After the associative inference test, participants were tested on their memory for $\mathrm{AB}$ and $\mathrm{BC}$ associations (direct test). Participants were informed that this test would be testing associations between objects that they directly studied in the study portion of the experiment (no inference necessary). Direct test trials were randomly intermixed with the condition that half of the $\mathrm{BC}$ associations were presented before their corresponding $\mathrm{AB}$ association with the shared $\mathrm{B}$ object, and the other half of the $\mathrm{BC}$ associations were presented after their corresponding AB association. This set-up controlled for any potential effect of testing of one association type on the other association type. As in the associative inference test, all direct test trials were three alternative-forced choice and self-paced. On AB test trials, participants were cued with an A object and had to choose the correct B object out of three displayed objects; the two foils were B objects that had been paired with a different A object than the cued A object. On BC test trials, participants were cued with a B object and had to choose the corresponding C object, 
and foils were $\mathrm{C}$ objects that had been paired with different B objects than the cued $\mathrm{B}$ object.

\section{Statistical analyses}

Accuracy was calculated as the proportion of correct test trials out of total trials (e.g., proportion of correct AB test trials out of total AB test trials). RT was calculated as the median RT out of correct test trials.

Associative inference performance. Mean accuracy and RT on the associative inference test was compared between presentation groups using independent samples ttests. Because of significant differences in accuracy between presentation groups on the direct test when outliers were included, I controlled for the effect of memory for AB and BC associations on associative inference performance. I conducted independent samples t-tests comparing presentation group associative inference accuracy and RT, only including associative inference trials for which the participant correctly answered both corresponding direct tests.

Direct test performance. Direct accuracy and RT were both analyzed via a 2 association type (AB, BC) x 2 presentation group (blocked, interleaved) mixed measures ANOVA. Follow-up comparisons were conducted for significant interactions. 


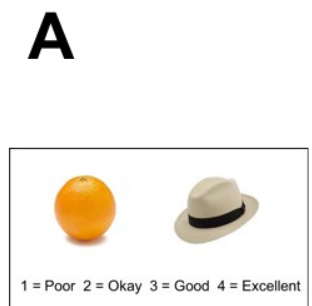

AB

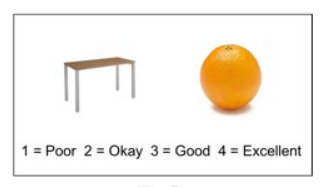

BC
B

Blocked Presentation

\begin{tabular}{|l|l|l|l|l|l|}
$\mathbf{A B}$ & $\mathbf{A B}$ & $\mathbf{A B}$ & $\mathbf{B C}$ & $\mathbf{B C}$ & $\mathbf{B C}$ \\
\hline
\end{tabular}

Interleaved Presentation

\begin{tabular}{|l|l|l|l|l|l|} 
AB & BC & AB & BC & AB & BC \\
\hline
\end{tabular}

\section{C}

Associative Inference

AC

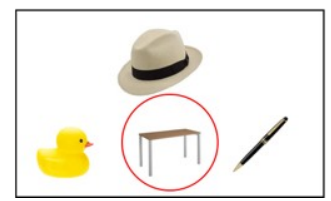

AB

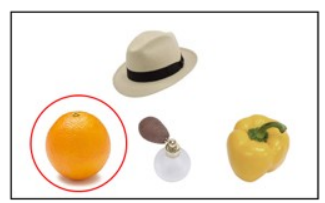

Direct

BC

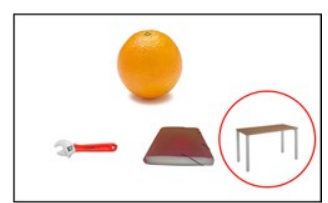

Figure 1. Overview of Experiment 1 design. A. During the study portion, participants were presented object-object associations, which each shared an object in common with another association (the orange in this case). B. Participants in the blocked presentation group saw three sets comprised of $\mathrm{AB}$ associations followed by three $\mathrm{BC}$ association sets. In the interleaved presentation group, participants saw alternating $A B$ and $B C$ sets. C. After the study portion, participants first completed an associative inference test, which required them to integrate across associations presented during the study. After the associative inference test, participants were tested on their memory for associations they viewed during the study portion (direct test). 


\section{Results: Experiment 1}

\section{Associative inference performance}

Mean accuracy of participants in each presentation group on the associative inference test is shown in Figure 2 below (outliers included: left; outliers excluded: right). Participants who saw blocked presentation of object associations (blocked presentation group) performed marginally better on the associative inference test than participants who viewed interleaved presentation (interleaved presentation group), but only when outliers were included (with outliers: $t(93)=-1.59, p=0.110$; without outliers: $t(87)=-1.00, p=0.310)$.
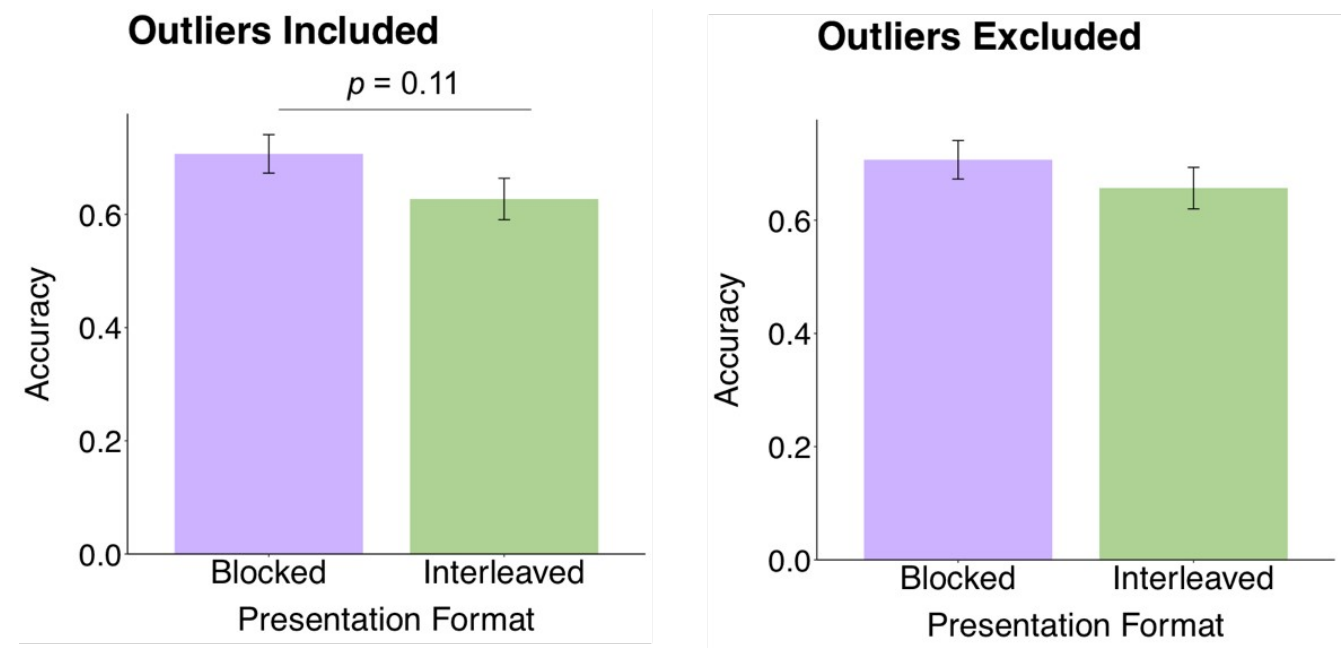

Figure 2. Accuracy on associative inference test. Participants in the blocked presentation group performed marginally better than participants in the interleaved group when outliers were included $\mathrm{p}<0.15$.

Because participants also differed significantly in direct test performance (Figure 4; outliers included: $F(1,91)=4.09, p=0.046, \eta^{2}=0.043$; outliers excluded: $F(1,87)=$ 1.05, $p=0.307, \eta_{P}^{2}=0.012$ ), differences in memory for individual associations may have driven associative inference performance differences. Thus, I controlled for 
differences in memory for individual associations between presentation groups, so that any differences in associative inference performance between presentation groups would be due to differences in the degree to which related information was integrated, the measure I was interested in. I controlled for memory for individual associations by only comparing associative inference trials for which participants correctly answered the corresponding direct test trials ( $\mathrm{AB}$ and $\mathrm{BC})$.

When considering associative inference test trials for which $\mathrm{AB}$ and $\mathrm{BC}$ were both learned (and remembered), participants in the blocked presentation group still performed marginally better on the associative inference test than participants in the interleaved presentation group (Figure 3; outliers included: $t(91)=-1.77, p=0.08$; outliers excluded: $t(87)=-1.18, p=0.24)$.

\section{Outliers Included}

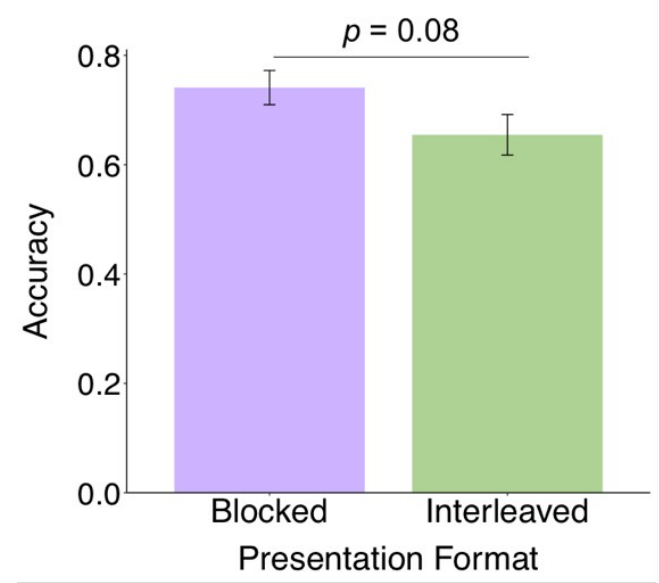

\section{Outliers Excluded}

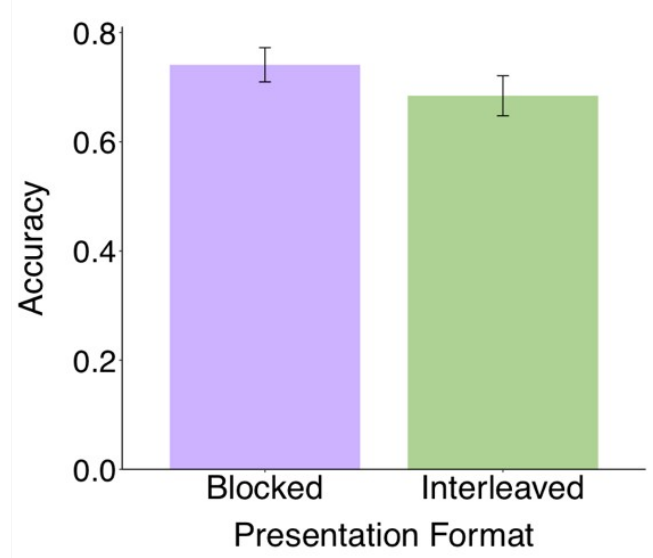

Figure 3. Accuracy on associative inference test trials for which participants correctly answered corresponding direct test trials. Participants in the blocked presentation group performed marginally better than participants in the interleaved group, but only when outliers were included $p<0.1$.

Mean reaction time (RT) on the associative inference test did not differ significantly between presentation groups, regardless of whether outliers were included or excluded 
(all $p>0.4$; Supplementary Figure S1). Because RT is highly variable across

individuals (Henmon and Wells, 1914; Jensen, 2006), this result is not too surprising.

\section{Direct test performance}

Accuracy

A 2 presentation group x 2 association type mixed measures ANOVA revealed a significant main effect of presentation group, but only when outliers were included (with outliers: $F(1,91)=4.09, p=0.046, \eta_{P}^{2}=0.043$; without outliers: $F(1,87)=1.05$, $p=0.307, \eta_{P}^{2}=0.012$ ), such that the participants in the blocked group had higher mean accuracy than participants in the interleaved group (Table 1; Figure 4, left). There was also a significant main effect of association type, regardless of outlier inclusion or exclusion (with outliers: $F(1,91)=12.46, p<0.001, \eta^{2}{ }_{P}=0.120$; without outliers: $F(1$, $\left.87)=2.03, p=0.002, \eta_{P}^{2}=0.101\right)$. That is, participants had significantly higher accuracy on $\mathrm{AB}$ tests than $\mathrm{BC}$ tests.

\begin{tabular}{|c|c|c|}
\hline \multicolumn{3}{|c|}{ OUTLIERS INCLUDED } \\
\hline & $\mathrm{AB}$ & $\mathrm{BC}$ \\
\hline Blocked & $0.889(0.125)$ & $0.874(0.163)$ \\
\hline Interleaved & $0.831(0.183)$ & $0.786(0.223)$ \\
\hline \multicolumn{3}{|c|}{ OUTLIERS EXCLUDED } \\
\hline & $\mathrm{AB}$ & $\mathrm{BC}$ \\
\hline Blocked & $0.889(0.125)$ & $0.874(0.163)$ \\
\hline Interleaved & $0.870(0.136)$ & $0.829(0.175)$ \\
\hline
\end{tabular}

Table 1. Mean accuracy (and SD) on direct tests 
Additionally, there was a marginal two-way presentation group $\mathrm{x}$ association type interaction when outliers were included (with outliers: $F(1,91)=3.18, p=0.078, \eta^{2}=$ 0.034; without outliers: $\left.F(1,87)=2.03, p=0.158 \eta^{2}=0.023\right)$. To investigate the twoway interaction, $\mathrm{AB}$ and $\mathrm{BC}$ accuracy differences within a given presentation group (e.g., blocked) were compared. Participants in the interleaved condition showed a significant $\mathrm{AB}$ vs. BC accuracy difference (outliers included: $t(47)=3.93, p<0.001$; outliers excluded: $t(42), p=0.002$; Figure 4, left). However, participants in the blocked condition did not show a significant accuracy difference $(t(44)=1.18, p=0.244)$, suggesting that this discrepancy between presentation groups drove the interaction.

\section{Reaction Time}

Some, but not at all, accuracy findings were recapitulated by analysis of RT data. A 2 presentation group x 2 association type mixed measures ANOVA revealed a significant main effect of association type (outliers included: $F(1,91)=43.88, p<$ 0.001, $\eta^{2}{ }_{P}=0.325$; outliers excluded: $\left.F(1,87)=43.11, p<0.001, \eta_{P}^{2}=0.331\right)$. Specifically, participants responded with significantly faster RT on AB test trials than BC test trials (Table 2; Figure 4, right). Because slower RT is associated with poorer learning (Craddock, Molet, and Miller, 2012), RT evidence complements findings of lower accuracy on BC test trials and further suggests that learning of and/or memory for $\mathrm{BC}$ associations is worse relative to learning of and/or memory for $\mathrm{AB}$ associations. 


\begin{tabular}{|c|c|c|}
\hline \multicolumn{3}{|c|}{ OUTLIERS INCLUDED } \\
\hline & $\mathrm{AB}$ & $\mathrm{BC}$ \\
\hline Blocked & $1.51(0.419)$ & $1.67(0.425)$ \\
\hline Interleaved & $1.50(0.414)$ & $1.73(0.522)$ \\
\hline \multicolumn{3}{|c|}{ OUTLIERS EXCLUDED } \\
\hline \multicolumn{3}{|c|}{$\mathrm{AB}$} \\
\hline Blocked & $1.51(0.419)$ & $\mathrm{BC}$ \\
\hline Interleaved & $1.55(0.378)$ & $1.79(0.425)$ \\
\hline
\end{tabular}

Table 2. Mean RT (and SD) on direct tests

There was no significant main effect of presentation group (outliers included: $F(1,91)=$ 0.0982, $p=0.755, \eta_{P}^{2}=0.001$; outliers excluded: $F(1,87)=0.843, p=0.361, \eta_{P}^{2}=$ 0.01), nor an interaction between presentation group and association type (outliers included: $F(1,91)=1.57, p=0.213, \eta_{P}^{2}=0.017$; outliers excluded: $F(1,87)=1.93, p=$ $\left.0.168, \eta_{P}^{2}=0.022\right)$.

As expected by absence of a significant presentation group $\mathrm{x}$ association type interaction, participants in both presentation groups had significantly slower RT on BC test trials (blocked: $t(44)=-3.32, p=0.002$; interleaved: $p<0.001$ both when outliers were included and excluded; Figure 4, right). This finding was in contrast to accuracy results, for which there was a significant difference in $A B$ and $B C$ accuracy in the interleaved presentation group only, resulting in a significant presentation group $\mathrm{x}$ association type interaction. Still, although the presentation group $\mathrm{x}$ association type RT interaction was not significant (outliers included: $F(1,91)=1.57, p=0.213, \eta^{2}=0.017$; outliers excluded: $F(1,87)=1.93, p=0.168, \eta^{2}=0.022$; Figure 4, right), the RT difference was numerically larger in the interleaved presentation group compared to blocked presentation group, regardless of whether outliers were included or excluded 
$\left(\mathrm{BC}_{\text {blocked }}-\mathrm{AB}_{\text {blocked }}=0.16 \mathrm{~s}\right.$ versus $\mathrm{BC}_{\text {interleaved }}-\mathrm{AB}_{\text {interleaved }}=0.23 \mathrm{~s}$ [with outliers] or

$\mathrm{BC}_{\text {interleaved }}-\mathrm{AB}_{\text {interleaved }}=0.24 \mathrm{~s}$ [without outliers]).
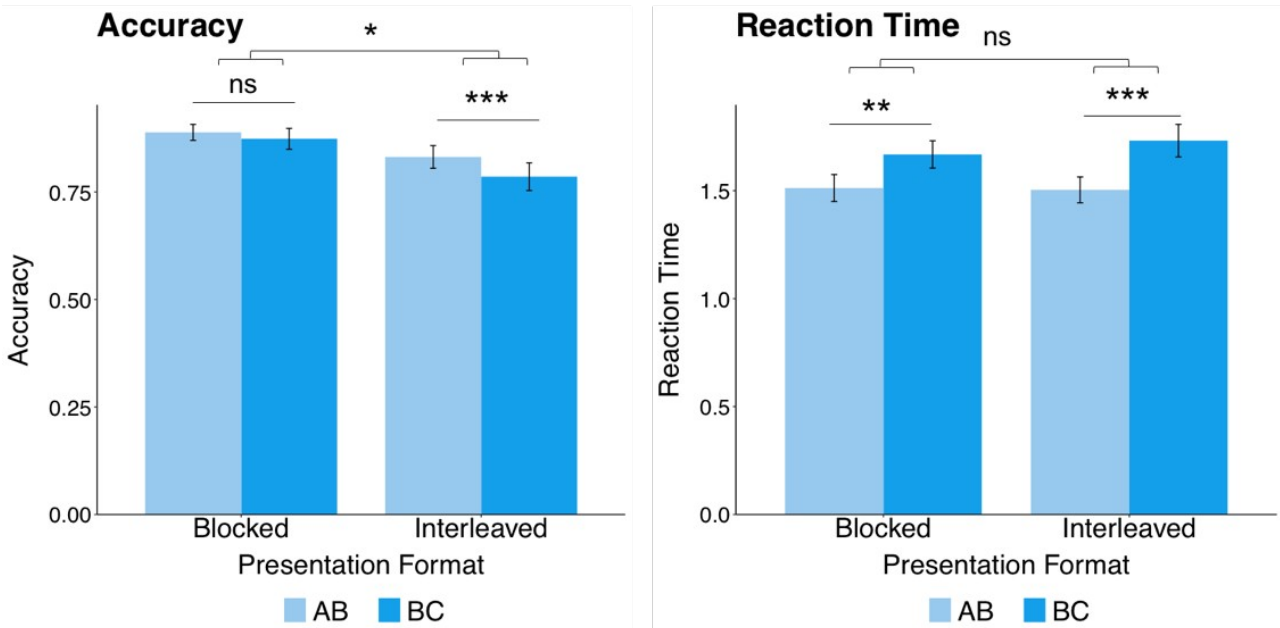

Figure 4. Mean accuracy and RT on direct tests, grouped by presentation group (outliers included). Participants in the blocked group did not have significantly different accuracy on the two direct tests and participants in interleaved presentation group performed with significantly worse accuracy on the BC test (*** denotes $p<0.001$ ), driving a significant main effect of association type. Despite having no accuracy differences between the two test types, participants in the blocked group performed with significantly slower RT on the BC test (** denotes $p<0.01$ ). Participants in the interleaved group also performed with significantly slower RT on the BC test (*** denotes $p<0.001$ ). 


\section{Discussion: Experiment 1}

\section{Integration of related associations (related information)}

My goal was to determine the effect of presentation format on memory integration. Differences in performance on an associative inference test between presentation groups was used to quantify the effect of presentation format on memory integration. I found partial evidence that participants in the blocked presentation group performed with higher accuracy on the associative inference test than participants in the interleaved presentation group. This marginally better accuracy was not accompanied by faster RT on the associative inference test in the blocked presentation group, but given the degree to which RT intrinsically varies across individuals (Henmon and Wells, 1914; Jensen, 2006), differences between presentation groups may have been difficult to detect.

However, accuracy differences on the associative inference test between presentation groups could also be explained by differences in memory for individual associations, rather than differences in tendency to successfully integrate, the measure I was interested in. After all, if individual associations are not remembered to begin with, participants would have no information to integrate. As such, a deficit in memory for individual associations could greatly impact performance on the associative inference test.

Because participants also differed significantly on direct test performance, memory for individual associations, and not the degree to which related associations were integrated, could potentially have driven the associative inference performance difference. I controlled for participants’ memory for individual associations by only 
considering associative inference trials for which participants correctly answered both corresponding $\mathrm{AB}$ and $\mathrm{BC}$ (direct) test trials. The marginal associative inference difference persisted, suggesting that enhanced associative inference performance in the blocked presentation group could not be entirely explained by better memory for individual associations and reflected, to some degree, better integration of related associations.

These results suggest that the strength of initial learning is more important than proximity in time to integration of related information. Although proximity in time has been shown to enhance neural signatures of integration and performance on tests requiring integration of related information (Zeithamova and Preston, 2017), participants in the interleaved presentation group, who viewed associations closer together in time than participants in the blocked presentation group, performed worse. Thus, the integration benefit from strongly establishing the initial association during blocked presentation (Zeithamova et al., 2012; Kuhl, et al., 2010; Schlichting and Preston, 2014; van Kesteren et al., 2018) surpassed any benefit of temporal proximity during interleaved presentation.

\section{Memory for related information (individual associations)}

There was some evidence that participants in the blocked presentation group also had better memory for individual associations, suggesting that both memory for and integration of related associations were enhanced by blocked presentation. These preliminary findings suggest that memory for related associations and integration of those associations might be mutually supportive memory processes and/or may be 
enhanced by the same learning condition factors, consistent with findings that integration of related information enhances (Anderson and McCulloch, 1999; Kuhl et al., 2010), rather than diminishes (Carpenter and Schacter, 2017; Tompary and Davachi, 2017), memory for related information. As with the associative inference test results, accuracy differences on the direct test were not accompanied by significant differences in RT between presentation groups.

In addition to comparing memory for associations between the presentation groups, I examined if memory for associations varied based on whether an association was presented in the first-encountered set ( $\mathrm{AB}$ associations) or in the secondencountered set (BC associations): presence of shared information across associations could either enhance learning of subsequent associations (facilitation; Kuhl et al., 2010; Schlichting and Preston, 2014; van Kesteren et al., 2018) or interfere with learning of subsequent associations (proactive interference; Underwood, 1949; Whitely, 1927; Postman, 1962). Additionally, I asked if the presentation formats differed based on the degree to which they produced facilitation or interference effects.

Accuracy data revealed no significant difference between AB and BC accuracy in the blocked presentation group, and significantly lower BC accuracy relative to AB accuracy in the interleaved presentation group. Because BC associations are the secondpresented associations, these accuracy findings suggest that the presentation formats differ in levels of proactive interference and/or the degree to which the learning of one piece of information promotes learning of a second, related piece of information (facilitation). 
That is, an absence of a decline in BC accuracy after blocked presentation, in contrast to a decline in BC accuracy after interleaved presentation, could be the result of less proactive interference and/or more facilitation during blocked presentation. Because participants in the blocked presentation group establish one set of information much more strongly than participants in the interleaved presentation group before being confronted with related information, we would expect reactivation of related information during new learning to be easier for participants in the blocked presentation group (Schlichting and Preston, 2014). Furthermore, reactivation of prior knowledge has been shown to facilitate learning of related information, making increased facilitation in the blocked presentation group consistent with prior findings (Kuhl et al., 2010; Schlichting and Preston, 2014; van Kesteren et al., 2018).

However, diminished proactive interference in the blocked presentation group relative to the interleaved presentation group would be contrary to previous findings (Postman, 1962; Del Missier, et al., 2018). Still, evidence of better integration in the blocked presentation group could explain a potential reduction in proactive interference: in addition to facilitating new learning (Anderson and McCulloch, 1999; Kuhl et al., 2010), integration has been shown to reduce interference (Chanales, et al., 2019). Thus, because blocked presentation also produced a marginal integration benefit, both reduced interference or facilitation in this presentation group would be consistent with integration findings and prior literature. Furthermore, the design of Experiment 1 left unclear whether increased facilitation or reduced interference explained the lack of an $\mathrm{AB} / \mathrm{BC}$ performance difference in this group. The design of Experiment 2, in turn, allowed us to distinguish between these two possibilities (see Methods: Experiment 2). 
When considering RT data, BC RT was slower relative to AB RT in both blocked and interleaved presentation groups. One explanation for a different pattern of results in the RT versus accuracy data might be that within-subject RT differences capture more subtle learning differences than accuracy (Christ et al., 2018). That is, BC learning may be impaired to some degree in both learning conditions, but the impairment is subtle enough to only manifest as a RT, and not accuracy, difference in the blocked presentation group.

However, because memory for individual associations was tested after the associative inference test, there was a potential for testing of inferred relationships across associations to worsen or enhance memory for the individual associations. Thus, we cannot make strong conclusions about differences in participants’ memory for individual associations between groups. Still, the implications of these findings motivate Experiment 2, which investigates memory for related associations in an unbiased way. 


\section{Methods: Experiment 2}

The findings of Experiment 1 suggested that blocked presentation may enhance both integration of and memory for studied information. However, because the direct test occurred after the associative inference test, performance on direct tests (our measure of memory for associations) could be biased. To further investigate the extent to which blocked presentation enhances memory for related information, in Experiment 2, memory for related associations was tested before the associative inference test.

To more completely characterize the potential effect of presentation format on memory for directly-studied, related information, two classes of associations were included in Experiment 2. Overlapping associations were identical to AB and BC associations in Experiment 1, except for the shared B element across associations was a face, rather than an object. In contrast, non-overlapping associations (UV and XY) consisted of object-face associations that did not share a face in common with any other associations in the study; UV associations were presented in the same block as AB associations and $\mathrm{XY}$ in the same block as BC.

Inclusion of overlapping and non-overlapping associations enabled Experiment 2 to address if shared information across associations produced facilitation and/or interference effects within a presentation group, and if these effects were differentially present in one presentation group over the other. Experiment 1 findings suggested that blocked presentation may produce greater facilitation and/or less proactive interference than interleaved presentation, but the experimental design could not distinguish between these two possibilities. We reasoned that any difference in performance between tests of $\mathrm{AB}$ and $\mathrm{BC}$ (overlapping) associations above and beyond any UV and XY (non- 
overlapping) performance difference would be due to the impact of overlapping information on memory for associations. Furthermore, the direction of the difference would determine whether overlapping information resulted in facilitation effects or interference effects. For example, if BC accuracy was higher than AB accuracy above and beyond any UV/XY accuracy, this would indicate a facilitation effect: In this case, memory for the $\mathrm{AB}$ association facilitated learning of a subsequent association which shared information, the BC association. Such a pattern of results would indicate facilitation, because the accuracy difference could not be explained by better memory for second-presented associations more generally, as the AB vs. BC accuracy difference would be above and beyond any UV vs. XY difference. If BC accuracy were lower than AB accuracy, above and beyond any UV/XY accuracy difference, this would indicate that presence of a shared element interfered with new learning (proactive interference effect). Finally, if $\mathrm{AB}$ accuracy was lower than BC accuracy, above and beyond any UV/XY accuracy difference, this would indicate that new learning interfered with memory for related information (retroactive interference effect).

Importantly, inclusion of UV vs. XY associations allowed for determination of whether observed effects were specific to overlapping associations, in which case the performance difference could be definitively attributed to presence of shared information.

\section{Participants}

104 volunteers participated in the experiment. Participants received research credit in their courses or monetary compensation for participation in the study. Three 
participants did not finish the study, and 16 were excluded due to a program error. Participants were determined to be outliers if their direct test accuracy was greater than 2.5 standard deviations from the mean: $\mathrm{n}=5$ participants were determined to be outliers. Because outlier inclusion or exclusion did not significantly impact the results, all subsequent analyses were performed with outliers included $(n=85)$.

\section{Materials}

Stimuli consisted of 126 faces and 168 everyday objects. Half of the faces $(n=$ 63) were female and the other half were male. Half of the faces $(n=63)$ were white and half of the faces were non-white $(n=63)$. Among non-white faces, one third $(n=21)$ were Asian, another third were black, and the final third were Latino. Within each racial group, the number of male and female faces was divided such that the remaining face after even division alternated as male or female across racial groups.

\section{Memory task}

Half of the objects (84) and half of the faces (42) were used to generate associations similar to those in Experiment 1: each association shared an element in common with one other association. However, unlike in Experiment 1, the associations were made up of face-object pairs and the shared element was a face. These face-object associations were part of the overlapping class. Experiment 2 included a new class of associations: non-overlapping associations. The other half of the objects (84) and remaining faces (84) were used to generate non-overlapping face-object associations, which did not share a face in common with any other associations. 
Similar to Experiment 1, associations were divided into two groups. Each of the two groups consisted of half non-overlapping associations (42) and half overlapping associations (42). Again, participants viewed six sets of associations with an opportunity to take a break between each set, and each set was comprised entirely of associations from one of the two groups. The group of associations presented in the first set (first-presented associations) were called AB (overlapping, as in Experiment 1) and UV (non-overlapping) associations. The group of associations presented second (second-presented associations) were called BC (overlapping, as in Experiment 1) and XY (non-overlapping) associations. See Figure 5A for a summary of the four association types.

Associations ( $\mathrm{AB}, \mathrm{BC}, \mathrm{UV}$, and $\mathrm{XY}$ ) were presented to the participants as in Experiment 1. Participants saw each of the 168 associations three times over the course of the study, the left/right position of all face-object pairs were randomized across trials, and participants were instructed to generate a unique story or mental image linking the face and object in the pair. As in Experiment 1, this task was designed to keep participants engaged in studying the associations, and was not analyzed for the purposes of the study. As in Experiment 1, before the actual study, participants ran through a practice version of the learning and rating task in which they viewed five $A B$ associations and five BC associations. As such, participants were aware that some of the associations in the study would contain overlapping information. No non-overlapping associations were included in the practice task. However, participants were just tested on their memory for the individual associations as a practice test, and did not complete an associative inference practice test. 
Like in Experiment 1, half of the participants saw all associations in an interleaved format, and half of the participants saw all associations in a blocked format. Each presentation set consisted of either all $\mathrm{AB}$ and $\mathrm{UV}$ associations, or all $\mathrm{BC}$ and $\mathrm{XY}$ associations. Participants in the blocked presentation group saw three repetitions of the group of $\mathrm{AB}$ and $\mathrm{UV}$ associations before three repetitions of the second group of associations (BC and $\mathrm{XY}$ associations) was presented. In the interleaved format, the two groups were alternated: the participant first saw $\mathrm{AB}$ and UV associations, followed by $\mathrm{BC}$ and $\mathrm{XY}$ associations, followed by $\mathrm{AB}$ and $\mathrm{UV}$ associations, etc. A summary of the two presentation groups is shown in Figure 5B. As in Experiment 1, breaks were given between each set of presented associations. $\mathrm{AB}$ and $\mathrm{UV}$ associations were randomly intermixed within each set with the constraint that the same $\mathrm{AB}$ associations, the same UV associations, or corresponding $\mathrm{AB}$ and $\mathrm{BC}$ associations (i.e., that shared their $\mathrm{B}$ object) were never presented back-to-back. The only potential place where these repetitions can occur is at the end of a presentation set, as the first association of the next set could be the same AB or UV association (if the next set is also comprised of $\mathrm{AB} / \mathrm{UV}$ associations) or a $\mathrm{BC}$ association with the same $\mathrm{B}$ face (if the set is a $\mathrm{BC} / \mathrm{XY}$ set). Face-object associations in BC/XY sets were randomly intermixed with the same constraint.

Following the study portion, participants were first tested on their memory for the associations, which differed from Experiment 1 where participants had to complete the associative inference test first. Participants completed the direct test first because the data on this test, instead of on the associative inference test, were used to compare the two presentation formats (blocked and interleaved) and the two classes of associations 
(overlapping and non-overlapping). This was because there could be no associative inference test of non-overlapping associations, and thus the two test classes (overlapping and non-overlapping) could not be compared using this measure.

Direct test presentations were counterbalanced by first randomly assigning a trial of each test type to a quartet, and then randomizing presentation of the $A B, B C$, $\mathrm{UV}$, and XY test trial within each quartet. The direct test was self-paced, meaning that the test advanced to the next trial once participants gave their response.

As in Experiment 1, participants were not informed of the overlap between the $\mathrm{AB}$ and $\mathrm{BC}$ associations at the start of the experiment. Following the direct test, participants were surprised with an AC associative inference test. Participants were informed that $\mathrm{A}$ and $\mathrm{C}$ objects that were paired with the same B face were indirectly related. In the associative inference three-alternative forced-choice test, participants were instructed to match an object at the top of the screen with the object below that was indirectly associated with that top object, based on what they had viewed during the study portion. As in the direct test, the associative inference test was self-paced, and participants viewed a $1 \mathrm{~s}$ fixation cross after giving their response.

\section{Statistical analyses}

As in Experiment 1, accuracy was calculated as the proportion of correct test trials out of total trials and RT was calculated as the median RT out of correct test trials.

Direct test performance. Direct test accuracy and RT were each analyzed first via a 2 presentation group (blocked, interleaved) x 2 association class (overlapping, non-overlapping) x 2 presentation order (first-presented, second-presented) mixed measures ANOVA. Follow-up comparisons were conducted for significant interactions 
and main effects. Despite no significant interactions with presentation group, 2 association class x 2 presentation order repeated measures ANOVAs were run in each presentation group separately to investigate the possibility that main effects of association class and presentation order were primarily driven by one presentation group over another.

Associative inference test performance. Mean accuracy and RT on the associative inference test was compared between presentation groups using independent samples t-tests. 


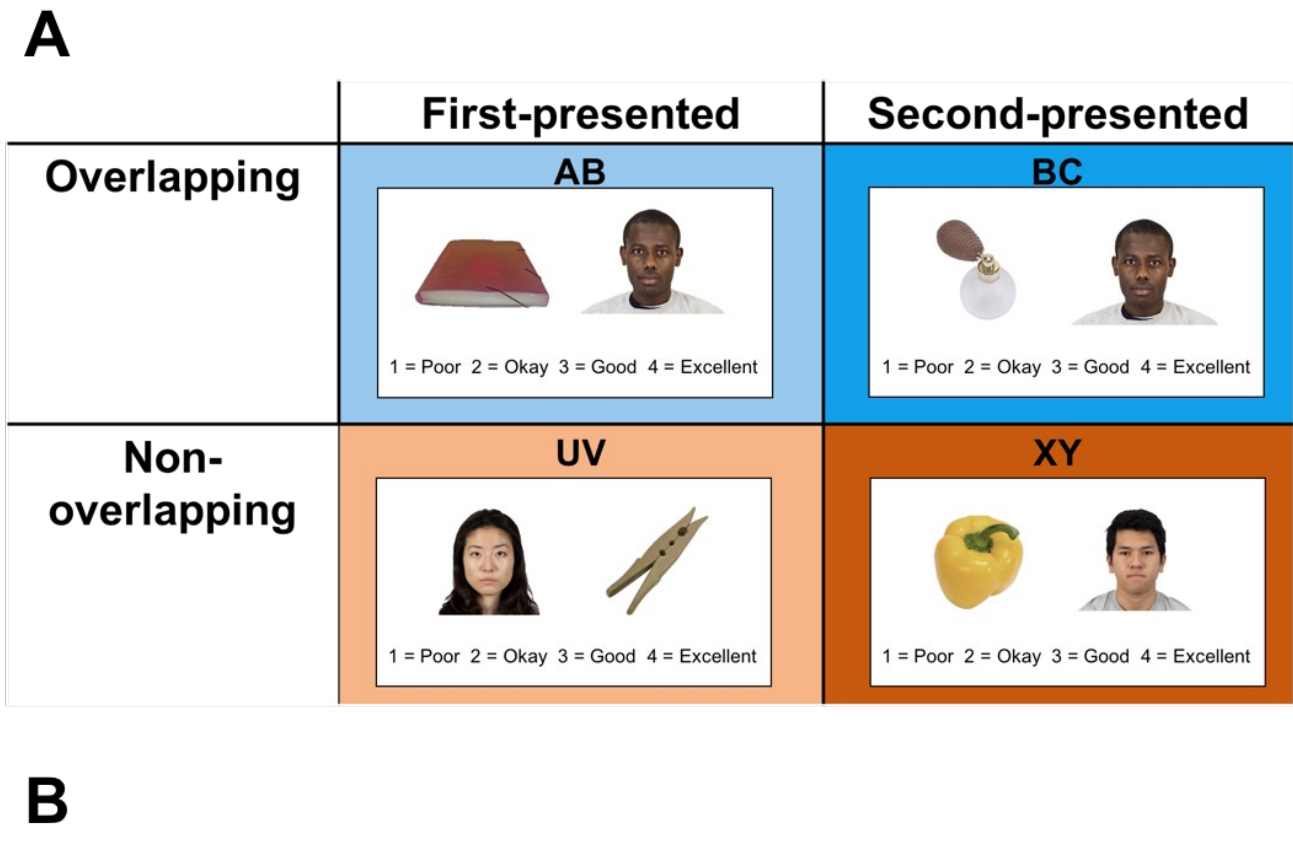

\section{Blocked Presentation} \begin{tabular}{|l|l|l|l|l|l|}
\hline AB /UV & $A B / U V$ & $A B / U V$ & $B C / X Y$ & $B C / X Y$ & $B C / X Y$ \\
\hline
\end{tabular}

\section{Interleaved Presentation}

\begin{tabular}{|l|l|l|l|l|l|}
\hline AB/UV & $B C / X Y$ & $A B / U V$ & $B C / X Y$ & $A B / U V$ & $B C / X Y$ \\
\hline
\end{tabular}

Figure 5. Overview of Experiment 2 design. A. Participants saw four different types of associations. The group of first-presented associations consisted of both overlapping (AB) and non-overlapping (UV) associations. The set of second-presented associations consisted of both overlapping (BC) and non-overlapping (XY) associations. B. Associations were presented in sets, each of which were comprised entirely of associations from one of the two groups. The sequence in which the two groups of associations were presented depended on a participants' presentation group. 


\section{Results: Experiment 2}

Inclusion or exclusion of outliers did not significantly impact results. Thus, results are shown here with outliers included, and the same results can be found in Supplementary Figures S3 (Accuracy) and S4 (RT) with outliers excluded. Because Experiment 2 tested memory for individual associations before the associative inference test, we could assess in an unbiased way the extent to which memory for individual associations was enhanced in one learning condition over another.

\section{Direct Tests: Accuracy}

A 2 presentation group x 2 association class x 2 presentation order (see Methods: Experiment 2) mixed measures ANOVA on accuracy data revealed a significant main effect of association class (i.e., overlapping vs. non-overlapping; $\left.F(1,83)=6.60, p=0.012, \eta_{P}^{2}=0.074\right)$, such that participants responded with higher accuracy on tests of overlapping associations compared to non-overlapping associations (Table 3; Figure 6; Table S1; Supplementary Figure S3). In addition to the main effect of association class, there was a significant main effect of presentation order $(F(1,83)=$ 5.46, $p=0.022, \eta_{P}^{2}=0.062$ ). Follow-up analyses demonstrated that participants had higher accuracy on tests of first-presented (i.e., AB and UV) associations compared to tests of second-presented (i.e., BC and XY) associations (Table 3; Figure 6; Table S1; Supplementary Figure S3). Importantly, there was no significant main effect of presentation group $\left(F(1,83)=0.993, p=0.753, \eta_{P}^{2}=0.001\right)$, meaning that direct accuracy did not significantly differ between presentation groups. 
Although there were no significant interactions with presentation group (all $F<$ $1.7, p>0.2$ ), I wanted to see if the main effects of association class and presentation order were primarily driven by accuracy data in one presentation group over the other. To investigate this possibility, a 2 association class $\mathrm{x} 2$ presentation order repeated measures ANOVA was run within each presentation group separately.

The 2 x 2 ANOVA did not reveal a significant main effect of presentation order in the interleaved presentation group $\left(F(1,41)=1.621, p=0.210, \eta^{2}=0.038\right.$; Table 3; Figure 6; Table S1; Supplementary Figure S3). Despite no main effect of presentation order, I was interested to see if there was a significant difference in specifically AB and BC accuracy in the interleaved presentation group, as in Experiment 1. A paired samples t-test revealed that, unlike in Experiment 1, there was no significant difference in accuracy between $\mathrm{AB}$ and $\mathrm{BC}$ tests $(t(41)=0.7289, p=0.470)$. However, there was a marginal main effect of presentation order in the blocked presentation group $(F(1,42)=$ 4.035, $\left.p=0.051, \eta_{P}^{2}=0.088\right)$, such that participants responded with higher accuracy on tests of first-presented associations relative to second-presented associations (Table 3; Figure 6; Table S1; Supplementary Figure S3). Finally, there were no significant interactions between presentation order and association class in either presentation group (all $F<0.8, p>0.35, \eta_{P}^{2}<0.02$ ).

The 2 x 2 ANOVA revealed a significant main effect of association class when run on data from participants in the interleaved presentation group $(F(1,41)=4.84, p=$ $\left.0.034, \eta_{P}^{2}=0.106\right)$, such that accuracy was higher on tests of overlapping associations relative to non-overlapping associations. This main effect was not significant for data from participants in the blocked presentation group $\left(F(1,42)=0.205, p=0.653, \eta^{2}{ }_{P}=\right.$ 
0.005). Thus, presence of shared information across associations enhances memory for the individual associations themselves during interleaved, but not blocked, presentation.

\begin{tabular}{|c|c|c|c|c|}
\hline & $\mathrm{AB}$ & $\mathrm{BC}$ & $\mathrm{UV}$ & $\mathrm{XY}$ \\
\hline Blocked & $0.884(0.161)$ & $0.854(0.184)$ & $0.881(0.166)$ & $0.838(0.192)$ \\
\hline Interleaved & $0.885(0.154)$ & $0.879(0.177)$ & $0.876(0.178)$ & $0.862(0.171)$ \\
\hline
\end{tabular}

Table 3. Mean accuracy (and SD) on direct tests

\section{Accuracy}

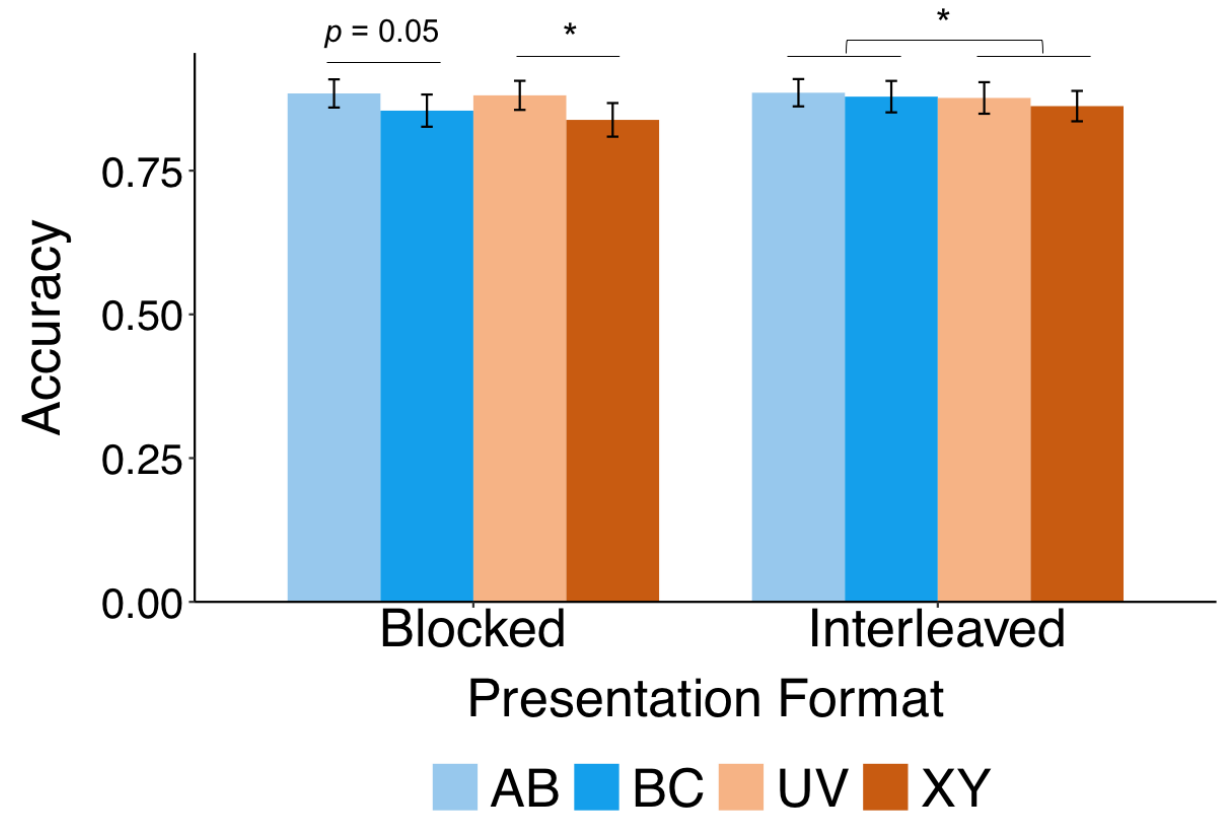

Figure 6. Mean accuracy on direct tests. Participants in the blocked presentation group performed significantly better on tests of second-presented associations, regardless of whether they were overlapping or non-overlapping, whereas participants in the interleaved presentation group performed better on tests of overlapping associations relative to non-overlapping associations. 


\section{Direct Tests: Reaction Time}

A 2 presentation group x 2 association class x 2 presentation order (see Methods: Experiment 2) mixed measures ANOVA on RT data had a significant main effect of association class $\left(F(1,83)=4.951, p=0.029, \eta^{2}{ }_{P}=0.056\right)$, such that participants responded with faster RT on tests of overlapping, compared to nonoverlapping, associations (Table 4; Figure 7; Table S2; Supplementary Figure S4). Unlike in the accuracy analysis, there was no significant main effect of presentation order $\left(F(1,83)=1.12, p=0.293, \eta_{P}^{2}=0.013\right.$; Supplementary Figure S4). There was no presentation group $\mathrm{x}$ association class interaction $\left(F(1,83)=0.916, p=0.341, \eta^{2}{ }_{P}=\right.$ 0.011), but there was a marginal presentation group x presentation order interaction $\left(F(1,83)=2.866, p=0.094, \eta_{P}^{2}=0.033\right.$; Supplementary Figure S4). However, this twoway interaction was qualified by a marginal three-way presentation group $\mathrm{x}$ association class x presentation order interaction $\left(F(1,83)=3.615, p=0.061, \eta^{2}=0.042\right.$; Figure Supplementary S4). Because of this three-way interaction, 2 presentation group x 2 association class mixed measures ANOVAs were conducted in each presentation group separately.

\begin{tabular}{|c|c|c|c|c|}
\hline & $\mathrm{AB}$ & $\mathrm{BC}$ & $\mathrm{UV}$ & $\mathrm{XY}$ \\
\hline Blocked & $1.92(0.490)$ & $1.94(0.466)$ & $1.96(0.456)$ & $1.97(0.420)$ \\
\hline Interleaved & $2.05(0.542)$ & $1.91(0.517)$ & $2.06(0.617)$ & $2.04(0.687)$ \\
\hline
\end{tabular}

Table 4. Mean RT (and SD) on direct tests 


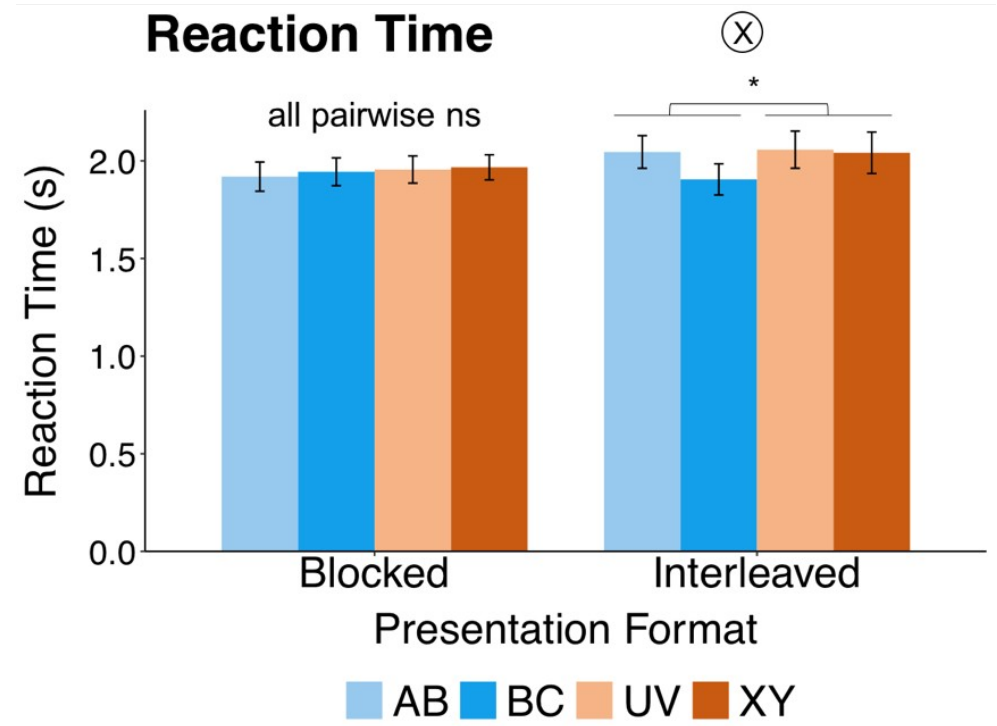

Figure 7. Mean RT on direct tests. Participants in the blocked presentation group did not have significantly different RT on any of the tests. In the interleaved presentation group, there was a significant association class $\mathrm{x}$ presentation order interaction $(p=$ 0.042), such that participants responded faster specifically on tests of overlapping, second-presented associations, indicating a potential facilitation effect.

The 2 x 2 ANOVA mixed measures ANOVA in the blocked presentation group revealed no significant main effects or interactions (all $F<1, p>0.3$; Figure 8, left; Supplementary Figure S4). In contrast, the 2 x 2 ANOVA mixed measures ANOVA in the interleaved presentation group revealed a significant main effect of association class $\left(F(1,41)=4.55, p=0.039, \eta_{P}^{2}=0.100\right.$; Supplementary Figure S4). Furthermore, there was a marginal main effect of presentation order $\left(F(1,41)=3.73, p=0.060, \eta_{P}^{2}=0.083\right.$; Supplementary Figure S4) and a significant association class x presentation order interaction $\left(F(1,41)=4.41, p=0.042, \eta^{2}=0.097\right.$; Figure 8, left; Figure S4) in the interleaved presentation group. To investigate this interaction further, RT on tests of first-presented and second-presented associations within an association class were compared via paired samples t-tests. RT on AB and BC (both overlapping) tests was significantly different $(t(41)=3.14, p=0.003)$ and RT on UV and XY tests was not 
significantly different $(t(41)=0.2862, p=0.776)$. Specifically, RT on second-presented associations was significantly faster than first-presented associations, but only when considering overlapping associations in the interleaved presentation group (Table 4; Table S2). The three-way interaction suggests that this specific effect of faster RT on second-presented, overlapping associations was unique to the interleaved presentation group.
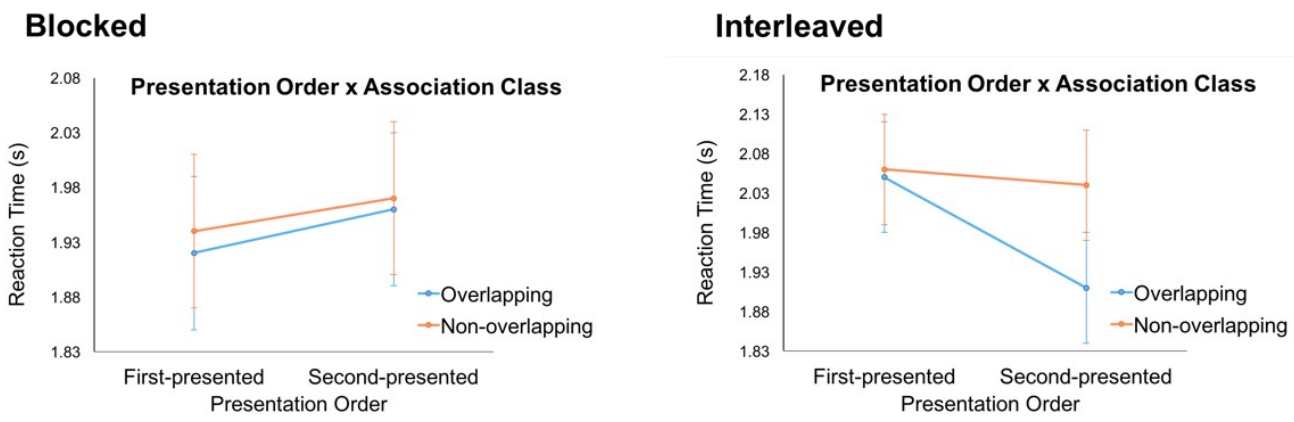

Figure 8. Effect of association class and presentation order on test RT. In the blocked presentation group (left) the two-way test class $\mathrm{x}$ order interaction was non-significant $(p=0.75)$, suggesting that differences in accuracy between first- and second-presented associations showed a similar pattern across classes (slower RT on tests of secondpresented associations, regardless of association class). In the interleaved presentation group (right) the two-way test class $\mathrm{x}$ order interaction was significant ( $p=0.042$ ). Specifically, the decrease in RT between tests of first-presented vs. second-presented associations was greater for overlapping than non-overlapping associations, suggesting that there might be a larger performance benefit on tests of second-presented associations for overlapping pairs.

\section{Associative Inference: Accuracy and Reaction Time}

Because the associative inference test occurred after the direct test, associative inference performance had the potential to be biased. Still, we considered if presentation group-differences in associative inference performance persisted after testing memory 
for individual associations. We found that associative inference performance did not differ between presentation groups, either in accuracy or RT (Accuracy: $t(83)=-0.213$, $p=0.832$, Supplementary Figure S5; RT: Welch’s t-test for unequal variances, $t(73.13)$ $=0.780, p=0.438$; Supplementary Figure S6). Thus, we did not replicate associative inference differences. 


\section{Discussion: Experiment 2}

\section{Memory for related information (individual associations)}

In Experiment 2, we were first interested in assessing differences in memory for associations between presentation formats in an unbiased way. Although in Experiment 1 we found that participants in the blocked presentation group had higher accuracy on the direct test, this test occurred after the associative inference test. Given that these participants also performed better on the associative inference test, this could have driven the performance difference. Thus, in Experiment 2, we tested memory for individual associations before the associative inference test.

Additionally, we were interested in more thoroughly characterizing the impact of presentation format on memory beyond overall differences in memory for associations between presentation groups. That is, we were interested in if one or both presentation formats produced facilitation or interference effects. It is unclear from the literature whether presence of shared information between associations would enhance learning of associations, in the case of facilitation (Kuhl et al., 2010; Schlichting and Preston, 2014; van Kesteren et al., 2018), or come at a cost to learning of associations, in the case of interference (Underwood, 1949; Whitely, 1927; Postman, 1962).

Furthermore, in Experiment 1, we found that participants in the blocked presentation group did not significantly differ in accuracy on $\mathrm{AB}$ and $\mathrm{BC}$ tests, but that participants in the interleaved presentation group had significantly worse accuracy on $\mathrm{BC}$ tests relative to $\mathrm{AB}$ tests. Such a finding suggested that the two presentation groups differ in levels of interference or facilitation. However, Experiment 1's design prevented us from determining whether this effect of equalized $\mathrm{AB}$ and $\mathrm{BC}$ accuracy in 
the blocked presentation group was due to less interference in the blocked presentation group compared to the interleaved presentation group (where BC accuracy was worse than AB accuracy), or due to more facilitation.

Inclusion of overlapping and non-overlapping associations in Experiment 2 allowed us to determine if facilitation or interference effects were present in one or both presentation groups. That is, any learning differences in non-overlapping pairs formed a baseline we could compare learning differences in overlapping pairs to. We reasoned that any learning differences in overlapping pairs, above and beyond the nonoverlapping pair baseline, would be due to facilitation or interference, with the direction of the difference determining which effect was observed.

In Experiment 2, we found no differences in memory for associations between the two presentation groups, regardless of whether associations were overlapping or non-overlapping. However, the presentation formats did differ in a more subtle way. That is, we found some evidence of facilitation in the interleaved, but not blocked, presentation group. Participants in the interleaved presentation group responded with faster RT only on tests of second-presented, overlapping (BC) associations, providing some evidence of better learning of this association type. Because participants did not significantly differ in RT on UV and XY (non-overlapping) tests in the interleaved presentation group, the effect cannot be explained by participants having faster RT on tests of second-presented associations in general. However, this specific effect on second-presented, overlapping associations did not produce a difference in accuracy: while participants performed better on tests of overlapping associations relative to nonoverlapping associations, the performance benefit did not interact with presentation 
order: participants performed with higher accuracy on tests of both first- and secondpresented overlapping associations.

Beyond whether the performance benefit was specific to second-presented, overlapping associations (as in facilitation) or to overlapping associations more generally, the accuracy and RT data both suggest that presence of shared information enhanced learning of overlapping associations. In the blocked presentation group, neither facilitation nor interference effects were observed, as accuracy and RT showed the same pattern of results, regardless of whether associations were overlapping or nonoverlapping. This result was surprising, given that strongly established information (as in blocked presentation) is more easily reactivated (Schlichting and Preston, 2014), and reactivation of related content, in turn, can facilitate new learning (Kuhl et al., 2010; Schlichting and Preston, 2014; van Kesteren et al., 2018).

Although blocked presentation did not produce facilitation or interference effects, there was an effect of presentation order. That is, participants in the blocked presentation group had lower accuracy and slower RT on tests of second-presented associations, regardless of whether associations were overlapping or non-overlapping. The effect of impaired accuracy on tests of second-presented associations in the blocked presentation group was unexpected for three main reasons. First, in Experiment 1, there were no significant differences between $\mathrm{AB}$ and $\mathrm{BC}$ accuracy in the blocked presentation group. Second, during blocked presentation participants view three repetitions of $\mathrm{BC} / \mathrm{XY}$ association blocks right before being tested on these associations. Third, this effect was exclusively seen in the blocked presentation group and not the 
interleaved presentation group, even though participants in the interleaved presentation group see just one consecutive block of BC/XY associations before the test.

Studies examining memory for both overlapping and non-overlapping verbal and non-verbal information have shown that participants have better memory for items they were recently exposed to than items they first learned (Knoedler, Hellwig, and Neath, 1999; Neath, 1993; Wright, et al., 1985; Pineño and Miller, 2005; Wheeler and Miller, 2007). Thus, lower accuracy on BC/XY tests relative to AB/UV tests is surprising. Still, one study by Morrison et al. (2014) demonstrated that task design can determine whether first-learned items are the best learned (primacy effects) or whether second-learned items are the best learned (recency effects): researchers could design a task for which participants showed greater primacy effects and a task for which participants showed greater recency effects. My finding that participants in the blocked presentation group demonstrated better learning of first-presented information suggests that blocked presentation may be an example of a task design that biases primacy effects, a possibility that should be investigated in future studies.

\section{Integration of related associations (related information)}

In Experiment 2, I did not replicate the Experiment 1 finding of marginally better associative inference performance in the blocked presentation group relative to the interleaved presentation group. There are two main possibilities that could explain this failure to replicate Experiment 1 associative inference findings in Experiment 2. First, testing participants' memory for individual associations before the associative inference test (as in Experiment 2) reminded participants in both presentation groups of 
shared information between associations, which could have equalized performance on the subsequent associative inference test. Second, face-object associations (Experiment 2) may be easier to integrate than object-object associations, again making the associative inference test easier for participants in both presentation groups (Experiment 1); too easy tasks will mask potential differences in learning between presentation groups, as participants in both presentation groups will be performing with high accuracy (ceiling effects), while tasks of sufficient difficulty will draw out any potential differences.

Although there is no way to directly test which possibility explains our inability to replicate marginal associative inference findings from Experiment 1, each has interesting implications. Both possible explanations (testing order and association stimuli) suggest that the integration was "easier" for participants in Experiment 2: when participants perform with high accuracy on the associative inference test overall, differences between groups can be obscured. Comparison of overall accuracy on the associative inference test (collapsing across presentation group) between the two experiments suggests this could be a possibility: Participants in Experiment 2 performed with an average accuracy of 0.79, whereas participants in Experiment 1 performed with an accuracy of $0.66(t(176)=3.88, p<0.001)$. 


\section{Discussion}

In this thesis, I examined the extent to which the sequence in which related information is presented impacts memory for and integration of that information. Participants viewed associations that shared elements in common in two different presentation formats (blocked and interleaved) and were tested on their memory for individual associations and on their ability to integrate associations via an associative inference test. I compared two presentation formats, blocked versus interleaved, which differed in the number of repetitions participants saw of one set of associations before being confronted with a second set of related associations. During blocked presentation, participants viewed three repetitions of the same set of associations before seeing the second set of associations; during interleaved presentation, sets of associations were alternated, meaning that participants viewed the first set of associations just once before viewing the second set of associations.

In Experiment 1, participants in the blocked presentation group performed marginally better on the associative inference test and significantly better on a test of their memory for individual associations. Still, despite these concurrent effects, better memory for individual associations in the blocked presentation group does not fully explain the associative inference performance benefit. This suggests that the associative inference performance difference truly reflects enhanced integration of related associations as a result of blocked presentation. Thus, strongly establishing one piece of information (as in blocked presentation), before being confronted with related information, strengthens memory for the information itself and promotes successful integration of the related information. 
The finding that blocked presentation provides some marginal benefit to both associative inference performance and memory for individual associations is surprising because interleaved presentation has been shown to be the superior presentation format for several types of learning (Carvalho and Goldstone, 2014; Rau, et al., 2013; Shea and Morgan, 1979). Still, researchers have suggested that blocked and interleaved presentation formats simply emphasize different aspects of learned information (Carvalho and Goldstone, 2015), making the superior learning format vary based on the demands of the task at hand.

In line with the proposal that blocked and interleaved presentation might enhance different features of the same learned information, Experiment 1 provided initial evidence that both memory for and integration of related information could be enhanced by blocked presentation. That is, blocked presentation meets the demands of a task that requires integration (associative inference test) and the demands of a task that requires retention of specific details of learned material (direct test) to a greater degree than interleaved presentation. The implication is that blocked presentation can promote integration across related information, without loss of memory for the original information itself. Studies have disagreed over whether increasing integration across learned, related material can lead to loss of memory for the originally learned material (Tompary and Davachi, 2017; Carpenter and Shacter, 2017), or better retention of the material (Anderson and McCulloch, 1999; Kuhl et al., 2010). The results of Experiment 1 suggest that increasing integration across learned material does not interfere with retention and/or initial learning of the material, and in fact might even enhance retention and/or initial learning. 
However, memory for individual associations was assessed after the associative inference test in Experiment 1, making performance on this test potentially biased. Thus, concrete conclusions cannot be made about the degree to which enhancing integration across related information might promote memory for the related information. In Experiment 2, memory for individual associations was assessed before the associative inference test, eliminating this potential for bias from our measure of memory for individual associations (direct test performance). In contrast to Experiment 1, no differences in performance on the direct test were observed between the two presentation groups (blocked versus interleaved).

Despite no between-group differences in memory for individual associations, the design of Experiment 2 allowed for a more nuanced analysis of memory for individual associations. Experiment 2 included two classes of associations: half of the associations shared an element in common with other associations (overlapping associations), and the other half did not share elements with any other associations (non-overlapping associations). Inclusion of overlapping and non-overlapping associations allowed us to determine the extent to which presence of shared information affected memory for individual associations in each presentation group. Although memory for overlapping and non-overlapping associations did not differ between presentation groups, within each group memory for associations could still vary based on association class (overlapping vs. non-overlapping). If there are differences in memory between association classes within a certain group, but no overall difference in memory for associations between the two groups, this could mean that both groups show the same 
pattern (e.g., better learning of overlapping compared to non-overlapping), or that the differences are too subtle to manifest as between-group differences.

Within-group comparisons revealed that overlapping associations were learned with significantly higher accuracy and significantly faster RT, both indicators of better learning, than non-overlapping associations in the interleaved group only. Furthermore, in the RT data we found specific evidence of a facilitation effect: participants responded with faster RT on tests of overlapping, second-presented associations, suggesting that learning of the $\mathrm{AB}$ association facilitates learning of the $\mathrm{BC}$ association. Thus, the presence of shared elements between associations enhances learning of associations when information is presented in interleaved. Importantly, blocked presentation did not produce facilitation effects nor interference effects: learning of overlapping and nonoverlapping associations was equivalent after blocked presentation. Thus, learning of overlapping associations is not hindered by interference in either presentation format, and is enhanced by, potentially, facilitation during interleaved presentation.

Interestingly, in Experiment 1, performance on the BC test drove direct test performance differences between presentation groups: AB test performance was not significantly different between presentation groups, but BC performance was significantly worse in the interleaved group. Thus, while Experiment 1 suggests a potential interference or absence of facilitation effect in the interleaved presentation group, Experiment 2 provides some evidence for a facilitation effect in the interleaved group.

Given these discrepancies, the effect of these presentation formats on memory for related information is not completely resolved by this thesis. However, the potential 
for bias in the direct test performance in Experiment 1 makes the direct learning findings of Experiment 2 more convincing, suggesting that there are facilitation effects as a result of interleaved, but not blocked, presentation. This result contradicts previous studies of facilitation, which indicate that strongly establishing the initial association and integrating associations produces facilitation (Kuhl et al., 2010; Schlichting and Preston, 2014; van Kesteren et al., 2018): Given that initial associations are more strongly established during blocked presentation and the behavioral (Experiment 1) and neural (Schlichting, Jeannete, and Preston, 2015) evidence of greater integration after blocked presentation, we would expect to observe facilitation effects in the blocked presentation group. Future experiments should more closely examine what factors, beyond strength of the initial association and integration of associations, could produce such facilitation effects in the interleaved presentation group.

The finding that blocked presentation does not result in a significant overlapping versus non-overlapping test performance difference, despite evidence that blocked presentation marginally promotes integration, is also surprising: Previous studies have indicated that integrating related episodes promotes better memory for the individual episodes themselves (Anderson and McCulloch, 1999; Kuhl et al., 2010). However, a study by Tompary and Davachi (2017) suggests that neural signatures of integration may only manifest with time, as memory integration relies on long-term memory processes. Because Experiment 2 tested memory for associations immediately after participants studied associations, potential long-term effects of enhanced integration across related associations on memory for the individual associations might only manifest with time. 
For example, in a study by Tompary and Davachi (2017), participants viewed non-overlapping and overlapping object-scene associations (similar to Experiment 2) and researchers examined neural representations when participants recalled the objectscene associations immediately after studying them and after a delay of the week. Researchers were examining how similar neural representations of non-overlapping associations were compared to neural representations of overlapping associations. The researchers found that the degree of the similarity was not significantly different immediately after encoding, but after a week, neural representations of overlapping associations were much more similar to each other than neural representations of nonoverlapping associations. Critically, the high degree of similarity between neural representations of overlapping associations after a week came at the loss of specific neural traces that uniquely represented each individual association, suggesting that integration of related information may worsen memory for each individual piece of information in the long term. Future studies could compare whether a delay of a week more negatively impacts overlapping direct test performance in one presentation format (e.g., blocked) relative to the other, and if this is the same presentation format that demonstrates evidence of enhanced memory integration.

Similarly, the time at which learning is assessed may influence which presentation format produces better performance. Studies have found that participants who practice mathematics problems (Taylor and Rohrer, 2010) and novel object categorization tasks (Calvharo and Goldstone, 2014) in blocked format perform better in the short-term, but that individuals who practice in interleaved ultimately perform better on tests delayed by one day (Taylor and Rohrer, 2010; Calvarharo and Goldstone, 
2014) and one week (Rohrer and Taylor, 2007). Taylor and Rohrer (2010) attribute the delayed benefit of interleaved practice to the difficulty of interleaved practice: Interleaved practice is more difficult than blocked practice because it requires participants to switch tasks, and thus participants cannot simply rely on memory of just practicing the same task directly before to complete subsequent practice repetitions. In the short term, this greater difficulty produces worse performance than blocked practice, but the difficulty of practice eventually prepares individuals better for a later test (Taylor and Rohrer, 2010). In my thesis, participants passively view associations and do not practice the eventual task (pairing one object with another). Thus, it would be interesting to see if the same effect would be seen for blocked versus interleaved presentation: that is, that blocked presentation produces better performance on the immediate test that requires integration of presented information (as reflected in the associative inference test results in this thesis), whereas interleaved presentation produces better performance on delayed tests of integration of learned material.

Furthermore, task difficulty can influence whether blocked versus interleaved practice produces better task performance. A study by Monteiro et al. (2017) tested whether blocked versus interleaved practice led to better electrocardiogram interpretation by medical students. In contrast to several findings in the literature (for review, see Dunlosky, et al., 2013), medical students in the blocked practice group performed better than students in the interleaved practice group on a 2-4 week delayed test. Because performance on the tests was overall very low, the researchers suggested that the added difficulty of interleaved practice to an already difficult task may have been detrimental. Similarly, a study by de Croock and van Merriënboer (2007) 
presented participants with malfunctions in a complex distiller system, and participants performed better on this difficult task after blocked presentation than interleaved. These studies demonstrate that task difficulty constitutes a boundary on the benefit derived from challenging interleaved practice. Future studies could increase the difficulty of the task, such as by using novel objects (rather than everyday objects), grayscale objects, and/or making the learning portion feedback-based, and examine how memory for and integration of related information is impacted in the short- and long-term.

Related to task difficulty, levels of prior knowledge about and/or practice with the task may affect whether blocked versus interleaved presentation/practice is most effective. Monteiro et al. (2017) note that medical students in the study had not previously learned about electrocardiogram interpretation in medical school, making students likely to be novices. In the study by de Croock and van Merriënboer (2007), participants were also unlikely to have much previous knowledge about how to fix distiller systems. In situations where individuals are confronted with difficult problems for which they have relatively little experience solving, interleaved practice may be unproductive. In fact, reviews have noted that initial exposures to a concept could be blocked, followed by interleaved practice (Rohrer, 2012; Schachter, 2014). Initial blocked exposure to difficult concepts could allow students to gain the necessary base knowledge to master the concept through more challenging interleaved practice. Given these predictions, future studies previously mentioned (i.e., including different levels of task difficulty and test timeframes) could also incorporate "hybrid" presentation formats to cover more presentation formats that might enhance memory for and integration of related information. 


\section{Conclusions}

\section{Memory for related information}

The experiments presented here do not fully resolve whether memory for and integration of related information would be optimized by the same or different learning conditions. Experiment 1 suggests that memory for and integration of related information are both promoted by blocked presentation. However, no overall memory differences were seen between the two presentation groups in Experiment 2. Furthermore, despite no memory differences between groups, interleaved presentation, but not blocked presentation, was shown to specifically enhance memory for associations that shared information with previously learned associations. Specifically, we found some evidence that interleaved presentation resulted in a facilitation effect: that is, learning one piece of information facilitated subsequent learning of realted information.

\section{Integration of related information}

The results of the experiments presented here suggest that blocked presentation may promote integration to a greater degree than interleaved presentation. This result is surprising, given that the majority of the literature has found interleaved presentation or training to result in better learning. However, I note that the benefit of blocked presentation may be related to when material was tested, and future studies should include delayed tests of integration and memory for related information to assess if this result persists, or is reversed. 


\section{Implications for instructional order}

More generally, the sequence in which information was presented was shown to modulate the impact shared information had on memory for studied material: presence of shared information resulted in better learning only after interleaved presentation. Furthermore, individuals integrated better across related information in the blocked presentation group, suggesting that instructional order impacts the degree to which participants integrate across related information. Thus, it is noteworthy that just changing the sequence in which information is presented to participants, without changing the total amount of time spent studying material, impacted learning. In an era of increased budget cuts (Leachman, et al., 2017; Edenfield, 2013) and, resultantly, research that aims to identify instruction methods that maximize students' learning with the least instruction time (Dolton, et al., 2003; Johnes, et al., 2017), these findings are particularly impactful. 


\section{Supplementary Figures}

\section{Experiment 1}
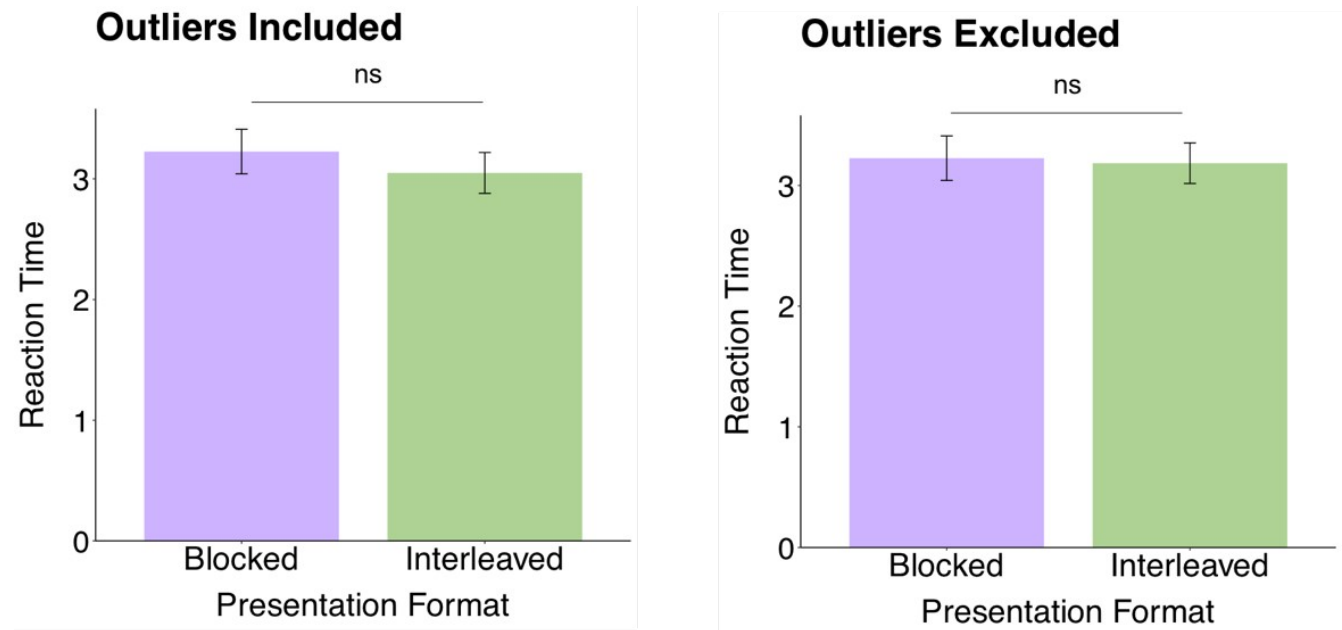

Figure S1. RT on associative inference tests. Presentation groups did not differ in associative inference test RT, regardless of whether outliers were included ( $p=0.48)$ or excluded $(p=0.87)$. 

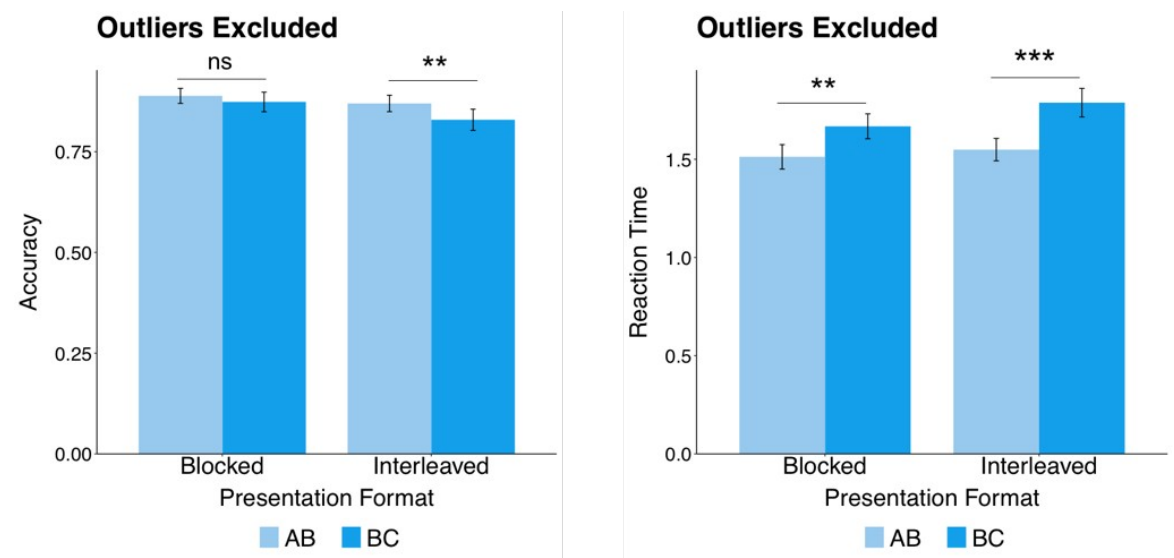

Figure S2. Mean accuracy and RT on direct tests, grouped by presentation group (outliers excluded). There was a significant main effect of association type, such that participants had both higher accuracy and faster RT on the AB test compared to the BC test. In the accuracy data, this was driven by significantly lower accuracy on the BC test relative to the $\mathrm{AB}$ test, whereas there was no difference in the blocked presentation group data. In the RT data, RT was significantly slower on the BC test in both the interleaved and blocked data. ( $* *$ denotes $\mathrm{p}<0.01$; $* *$ denotes $\mathrm{p}<0.001$ ). 


\section{Experiment 2}

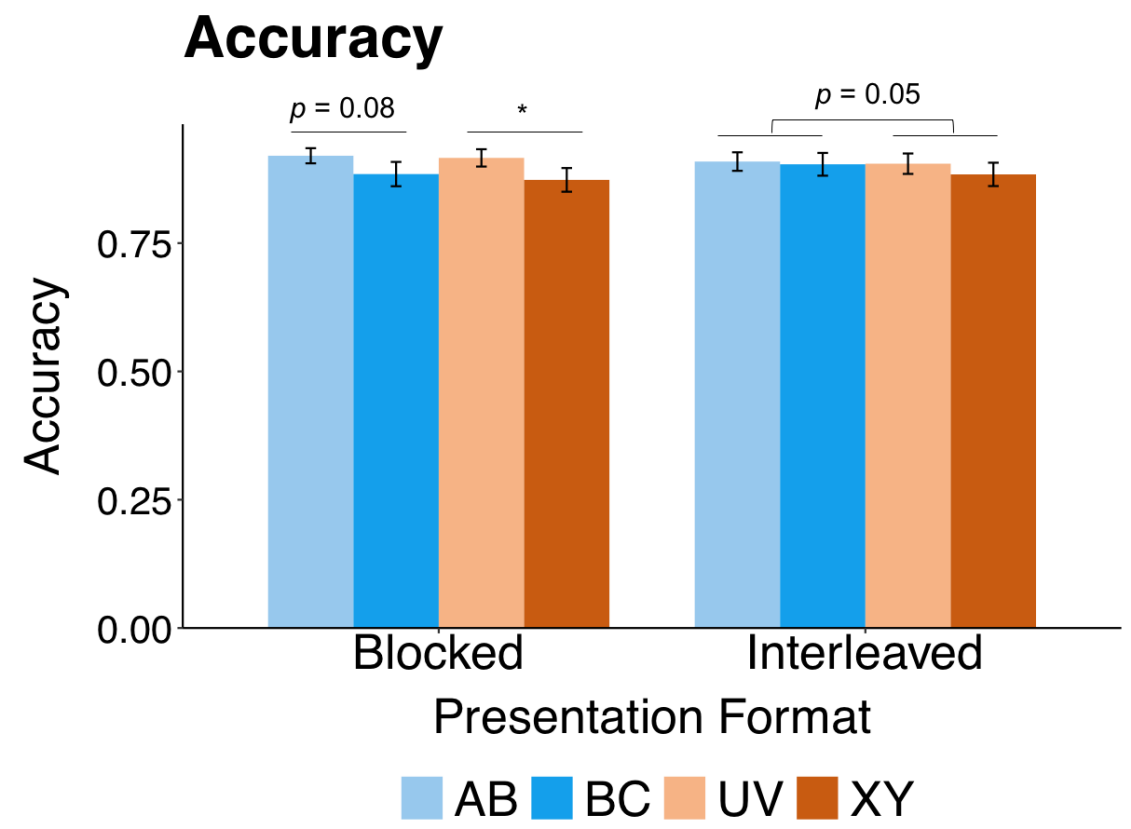

Figure S3. Mean accuracy on direct tests (outliers excluded). A three-way presentation group $\mathrm{x}$ presentation order $\mathrm{x}$ association class ANOVA revealed significant main effects of association class ( $p=0.029)$, presentation order $(p=0.015)$, and no other significant main effects or interactions (all $p>0.18$ ). In the blocked presentation group, there was a significant main effect of presentation order $(p=0.049)$, but no other significant main effects or interactions, as in the data with outliers included (all $p>$ $0.24)$. The interleaved presentation group findings mostly recapitulated findings when outliers were included: there was a marginal main effect of association class ( $p=$ $0.053)$, a marginal main effect of presentation order $(p=0.128)$, and a marginal association class $\mathrm{x}$ presentation order interaction $(p=0.156)$. 


\section{Reaction Time}

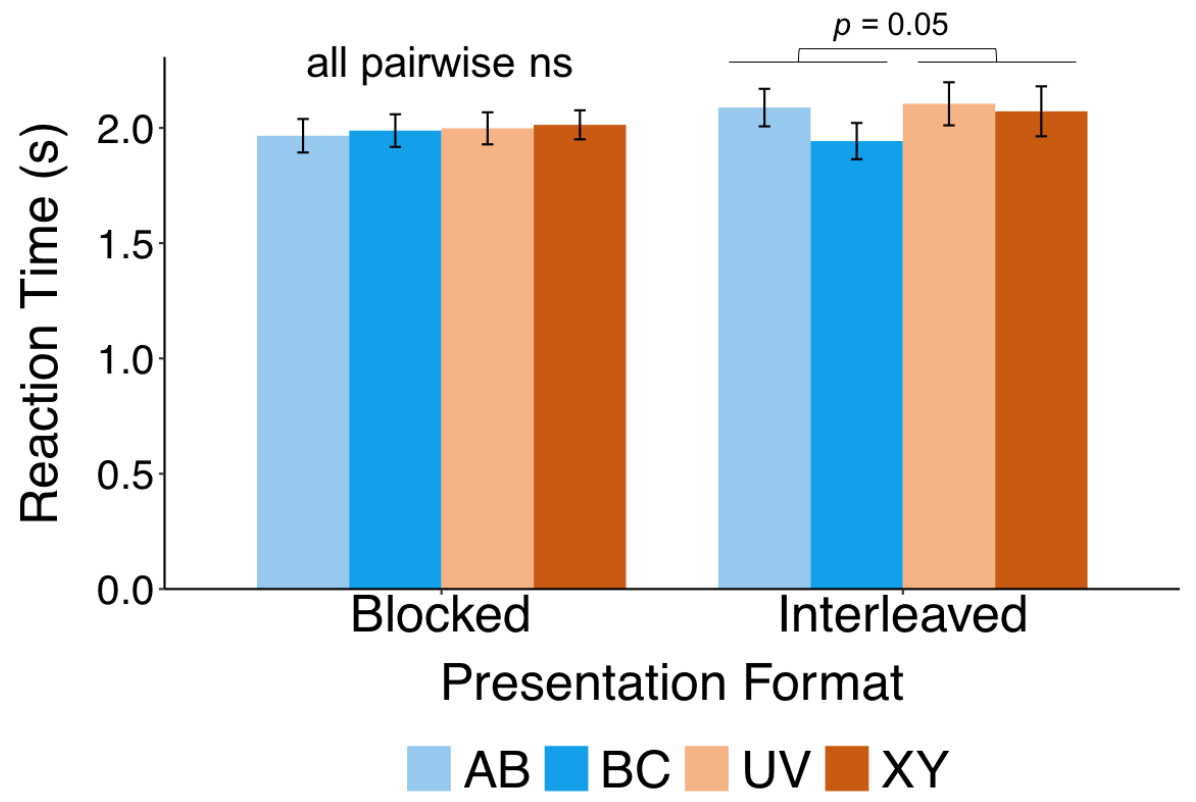

Figure S4. Mean RT on direct tests (outliers excluded). A three-way presentation group $\mathrm{x}$ presentation order $\mathrm{x}$ association class ANOVA revealed a significant main effect of association class $(p=0.04)$ and again marginal two-way presentation order $\mathrm{x}$ presentation group ( $p=0.072$, which was qualified by a marginal three-way interaction $(p=0.166)$. When considering the blocked presentation group data, there were again no significant main effects or interactions (all $p>0.35$ ). The interleaved presentation group showed a similar pattern of results as when outliers were included: there was a marginal main effect of association class $(p=0.053)$, a significant main effect of presentation order $(p=0.039)$, and a marginal association class $\mathrm{x}$ presentation order interaction $(p=0.075)$. 

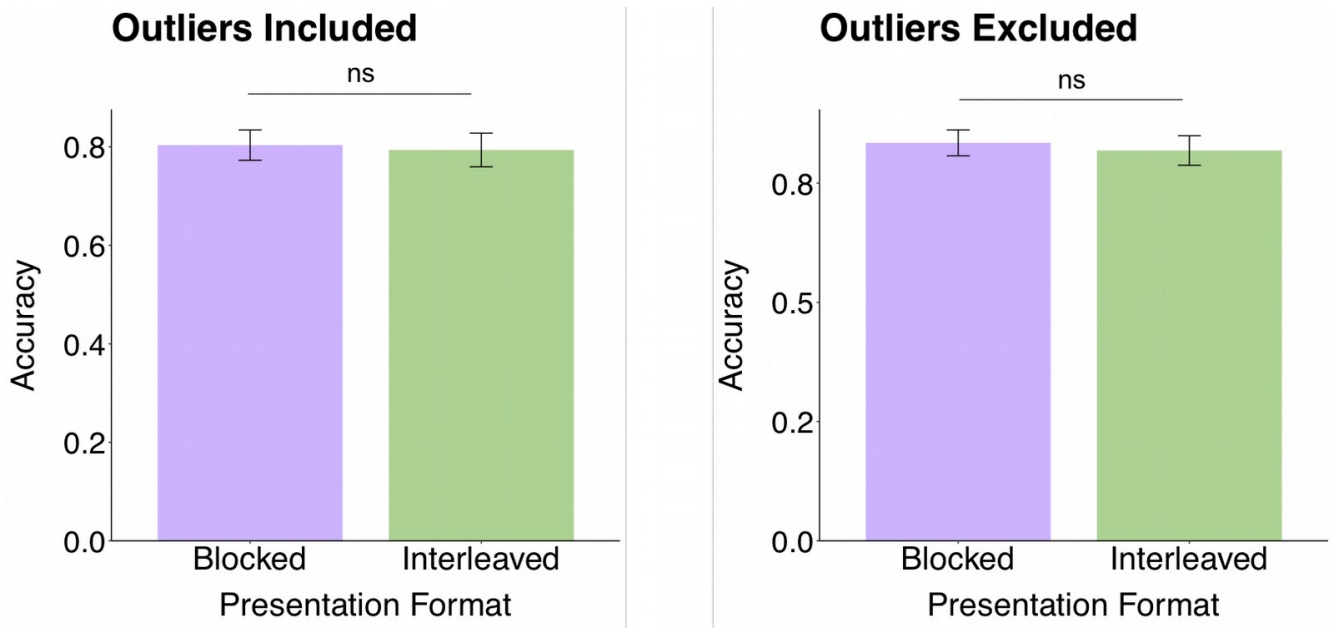

Figure S5. Associative inference test accuracy. The two presentation groups did not significantly differ on associative inference test accuracy, regardless of whether outliers were included or excluded (outliers included: $t(83), p=0.83$; outliers excluded: $t(78), p$ $=0.69$ ).

Outliers Included

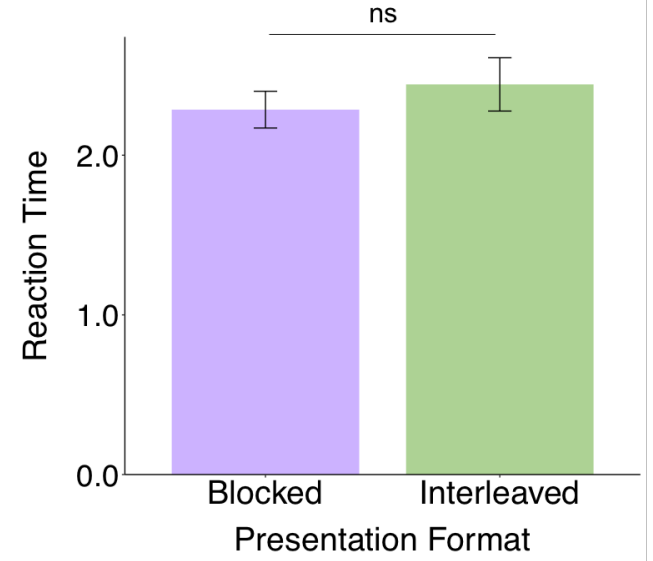

Outliers Excluded

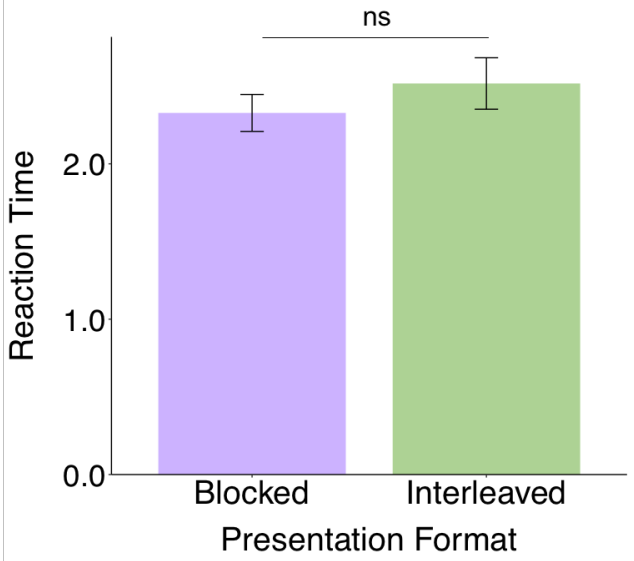

Figure S6. Associative inference test RT. The two presentation groups did not significantly differ on associative inference test RT, regardless of whether outliers were included or excluded (outliers included: $t(83), p=0.55$; outliers excluded: $t(78), p=$ $0.56)$. 


\section{Supplementary Tables}

\begin{tabular}{|c|c|c|c|c|}
\hline & $\mathrm{AB}$ & $\mathrm{BC}$ & $\mathrm{UV}$ & $\mathrm{XY}$ \\
\hline Blocked & $0.920(0.093)$ & $0.885(0.15)$ & $0.916(0.107)$ & $0.873(0.146)$ \\
\hline Interleaved & $0.909(0.114)$ & $0.904(0.140)$ & $0.905(0.126)$ & $0.884(0.114)$ \\
\hline
\end{tabular}

Table S1. Mean accuracy (and SD) on direct tests (outliers excluded)

\begin{tabular}{|c|c|c|c|c|}
\hline & $\mathrm{AB}$ & $\mathrm{BC}$ & $\mathrm{UV}$ & $\mathrm{XY}$ \\
\hline Blocked & $1.97(0.460)$ & $1.99(0.448)$ & $2.00(0.439)$ & $2.01(0.398)$ \\
\hline Interleaved & $2.09(0.515)$ & $1.94(0.497)$ & $2.10(0.590)$ & $2.07(0.686)$ \\
\hline
\end{tabular}

Table S2. Mean RT (and SD) on direct tests (outliers excluded) 


\section{Bibliography}

Anderson, M. C., \& McCulloch, K. C. (1999). Integration as a General Boundary Condition on Retrieval-Induced Forgetting. Journal of Experimental Psychology: Learning, Memory, and Cognition, 25(3), 608-629. 22.

Baker, D. P., Fabrega, R., Galindo, C., \& Mishook, J. (2004). Instructional Time and National Achievement: Cross-National Evidence. PROSPECTS, 34(3), 311-334. https://doi.org/10.1007/s11125-004-5310-1

Carpenter, A. C., \& Schacter, D. L. (2017). Flexible retrieval: When true inferences produce false memories. Journal of Experimental Psychology: Learning, Memory, and Cognition, 43(3), 335-349. https://doi.org/10.1037/xlm0000340

Carpenter, S. K. (2014). Spacing and Interleaving of Study and Practice. 11.

Carvalho, P. F., \& Goldstone, R. L. (2014). Effects of interleaved and blocked study on delayed test of category learning generalization. Frontiers in Psychology, 5, 111. https://doi.org/10.3389/fpsyg.2014.00936

Carvalho, P. F., \& Goldstone, R. L. (2015). The benefits of interleaved and blocked study: Different tasks benefit from different schedules of study. Psychonomic Bulletin \& Review, 22(1), 281-288. https://doi.org/10.3758/s13423-014-0676-4

Cepeda, N. J., Pashler, H., Vul, E., Wixted, J. T., \& Rohrer, D. (2006). Distributed practice in verbal recall tasks: A review and quantitative synthesis. Psychological Bulletin, 132(3), 354-380. https://doi.org/10.1037/00332909.132.3.354

Chanales, A. J. H., Dudukovic, N. M., Richter, F. R., \& Kuhl, B. A. (2019). Interference between overlapping memories is predicted by neural states during learning. Nature Communications, 10(1), 5363. https://doi.org/10.1038/s41467-01913377-x

Christ, B. U., Combrinck, M. I., \& Thomas, K. G. F. (2018). Both Reaction Time and Accuracy Measures of Intraindividual Variability Predict Cognitive Performance in Alzheimer's Disease. Frontiers in Human Neuroscience, 12, 111. https://doi.org/10.3389/fnhum.2018.00124

Craddock, P., Molet, M., \& Miller, R. R. (2012). Reaction time as a measure of human associative learning. Behavioural Processes, 90(2), 189-197. https://doi.org/10.1016/j.beproc.2012.01.006 
Croock, M., \& Van Merrienboer, J. J. G. (2007). Paradoxical effects of information presentation formats and contextual interference on transfer of a complex cognitive skill. Computers in Human Behavior, 23, 1740-1761. https://doi.org/10.1016/j.chb.2005.10.003

Del Missier, F., Sassano, A., Coni, V., Salomonsson, M., \& Mäntylä, T. (2018). Blocked vs. Interleaved presentation and proactive interference in episodic memory. Memory, 26(5), 697-711. https://doi.org/10.1080/09658211.2017.1402937

Dolton, P., Marcenaro, O. D., \& Navarro, L. (2003). The effective use of student time: A stochastic frontier production function case study. Economics of Education Review, 22(6), 547-560. https://doi.org/10.1016/S0272-7757(03)00027-X

Dunlosky, J., Rawson, K. A., Marsh, E. J., Nathan, M. J., \& Willingham, D. T. (2013). Improving Students’ Learning With Effective Learning Techniques: Promising Directions From Cognitive and Educational Psychology. Psychological Science in the Public Interest, 14(1), 4-58. https://doi.org/10.1177/1529100612453266

Edenfield, B. D. (n.d.). Teacher Perceptions of the Impact of Reduced School Budgets on Their Ability to Meet Instructional Needs of Their Students. 149.

Henmon, V. A. C., \& Wells, F. L. (1914). Concerning individual differences in reaction times. Psychological Review, 21(2), 153-156. https://doi.org/10.1037/h0073142

Hulbert, J. C., \& Anderson, M. C. (2020). Does retrieving a memory insulate it against memory inhibition? A retroactive interference study. Memory, 28(3), 293-308. doi: 10.1080/09658211.2019.1710216

Hupbach, A. (2011). The Specific Outcomes of Reactivation-Induced Memory Changes Depend on the Degree of Competition between Old and New Information. Frontiers in Behavioral Neuroscience, 5, 33. https://doi.org/10.3389/fnbeh.2011.00033

Janiszewski, C., Noel, H., \& Sawyer, A. G. (2003). A Meta-analysis of the Spacing Effect in Verbal Learning: Implications for Research on Advertising Repetition and Consumer Memory. Journal of Consumer Research, 30(1), 138-149. https://doi.org/10.1086/374692

Jensen, A. R. (2007). Clocking the mind: Mental chronometry and individual differences. Amsterdam: Elsevier. (n.d.).

Johnes, J., Portela, M., \& Thanassoulis, E. (2017). Efficiency in education. Journal of the Operational Research Society, 68(4), 331-338. https://doi.org/10.1057/s41274-016-0109-z 
Knoedler, A. J., Hellwig, K. A., \& Neath, I. (1999). The shift from recency to primacy with increasing delay. Journal of Experimental Psychology: Learning, Memory, and Cognition, 25(2), 474-487. https://doi.org/10.1037/0278-7393.25.2.474

Koen, J. D., \& Rugg, M. D. (2016). Memory Reactivation Predicts Resistance to Retroactive Interference: Evidence from Multivariate Classification and Pattern Similarity Analyses. Journal of Neuroscience, 36(15), 4389-4399. https://doi.org/10.1523/JNEUROSCI.4099-15.2016

Kuhl, B. A., Shah, A. T., DuBrow, S., \& Wagner, A. D. (2010). Resistance to forgetting associated with hippocampus-mediated reactivation during new learning. Nature Neuroscience, 13(4), 501-506. https://doi.org/10.1038/nn.2498

Leachman, M., Masterson, K., \& Figueroa, E. (n.d.). A Punishing Decade for School Funding. 17.

McAllister, E. W., \& Ley, R. (1972). Effects of spaced presentations of stimulus terms, response terms, and stimulus-response pairs on retention in paired-associate learning. Journal of Experimental Psychology, 94(1), 68-73. https://doi.org/10.1037/h0032775

Monteiro, S., Melvin, L., Manolakos, J., Patel, A., \& Norman, G. (2017). Evaluating the effect of instruction and practice schedule on the acquisition of ECG interpretation skills. Perspectives on Medical Education, 6(4), 237-245. https://doi.org/10.1007/s40037-017-0365-x

Neath, I. (1993). Distinctiveness and serial position effects in recognition. Memory \& Cognition, 21(5), 689-698. https://doi.org/10.3758/BF03197199

Pineño, O., \& Miller, R. R. (2005). Primacy and recency effects in extinction and latent inhibition: A selective review with implications for models of learning. Behavioural Processes, 69(2), 223-235. https://doi.org/10.1016/j.beproc.2005.02.006

Postman, L. (1962). Transfer of training as a function of experimental paradigm and degree of first-list learning. Journal of Verbal Learning and Verbal Behavior, 1(2), 109-118. https://doi.org/10.1016/S0022-5371(62)80007-3

Rau, M. A., Aleven, V., \& Rummel, N. (2013). Interleaved practice in multidimensional learning tasks: Which dimension should we interleave? Learning and Instruction, 23, 98-114. https://doi.org/10.1016/j.learninstruc.2012.07.003

Rohrer, D. (2012). Interleaving Helps Students Distinguish among Similar Concepts. Educational Psychology Review, 24(3), 355-367. https://doi.org/10.1007/s10648-012-9201-3 
Rohrer, D., \& Taylor, K. (2007). The shuffling of mathematics problems improves learning. Instructional Science, 35(6), 481-498. https://doi.org/10.1007/s11251007-9015-8

Schlichting, M. L., \& Preston, A. R. (2014). Memory reactivation during rest supports upcoming learning of related content. Proceedings of the National Academy of Sciences, 111(44), 15845-15850. https://doi.org/10.1073/pnas.1404396111

Schlichting, Margaret L., Mumford, J. A., \& Preston, A. R. (2015). Learning-related representational changes reveal dissociable integration and separation signatures in the hippocampus and prefrontal cortex. Nature Communications, 6(1), 8151. https://doi.org/10.1038/ncomms9151

Schlichting, Margaret L, \& Preston, A. R. (2015). Memory integration: Neural mechanisms and implications for behavior. Current Opinion in Behavioral Sciences, 1, 1-8. https://doi.org/10.1016/j.cobeha.2014.07.005

Schlichting, Margaret L., Zeithamova, D., \& Preston, A. R. (2014). CA1 subfield contributions to memory integration and inference. Hippocampus, 24(10), 12481260. https://doi.org/10.1002/hipo.22310

Shea, J. B., \& Morgan, R. L. (1979). Contextual interference effects on the acquisition, retention, and transfer of a motor skill. Journal of Experimental Psychology: Human Learning \& Memory, 5(2), 179-187. https://doi.org/10.1037/02787393.5.2.179

Shing, Y. L., \& Brod, G. (2016). Effects of Prior Knowledge on Memory: Implications for Education: Prior Knowledge, Memory, Brain, and Education. Mind, Brain, and Education, 10(3), 153-161. https://doi.org/10.1111/mbe.12110

Shohamy, D., \& Wagner, A. D. (2008). Integrating Memories in the Human Brain: Hippocampal-Midbrain Encoding of Overlapping Events. Neuron, 60(2), 378389. https://doi.org/10.1016/j.neuron.2008.09.023

Taylor, K., \& Rohrer, D. (2010). The effects of interleaved practice. Applied Cognitive Psychology, 24(6), 837-848. https://doi.org/10.1002/acp.1598

Tompary, A., \& Davachi, L. (2017). Consolidation Promotes the Emergence of Representational Overlap in the Hippocampus and Medial Prefrontal Cortex. Neuron, 96(1), 228-241.e5. https://doi.org/10.1016/j.neuron.2017.09.005

Underwood, B. J. (1949). Proactive inhibition as a function of time and degree of prior learning. Journal of Experimental Psychology, 39(1), 24-34.

https://doi.org/10.1037/h0059550 
van Kesteren, Marlieke Tina Renée, Krabbendam, L., \& Meeter, M. (2018). Integrating educational knowledge: Reactivation of prior knowledge during educational learning enhances memory integration. NPJ Science of Learning, 3(11), 1-8. https://doi.org/10.1038/s41539-018-0027-8

Verde, M. F. (2004). Associative interference in recognition memory: A dual-process account. Memory \& Cognition, 32(8), 1273-1283.

https://doi.org/10.3758/BF03206318

Wheeler, D. S., \& Miller, R. R. (2007). Primacy effects induced by temporal or physical context shifts are attenuated by a preshift test trial. The Quarterly Journal of Experimental Psychology, 60(2), 191-210. https://doi.org/10.1080/17470210600790240

Whitely, P. L. (1927). The dependence of learning and recall upon prior intellectual activities. Journal of Experimental Psychology, 10(6), 489-508. https://doi.org/10.1037/h0075186

Wright, A. A. (1994). Primacy effects in animal memory and human nonverbal memory. Animal Learning \& Behavior, 22(2), 219-223. https://doi.org/10.3758/ BF03199923

Zeithamova, Dagmar, Dominick, A. L., \& Preston, A. R. (2012). Hippocampal and Ventral Medial Prefrontal Activation during Retrieval-Mediated Learning Supports Novel Inference. Neuron, 75(1), 168-179. https://doi.org/10.1016/j.neuron.2012.05.010

Zeithamova, Dagmar, \& Preston, A. R. (2017). Temporal Proximity Promotes Integration of Overlapping Events. Journal of Cognitive Neuroscience, 29(8), 1311-1323. https://doi.org/10.1162/jocn_a_01116 